\title{
Orthogonal Structure on a Wedge and on the Boundary of a Square
}

\author{
Sheehan Olver $^{1} \cdot$ Yuan $X u^{2}$
}

Received: 24 October 2017 / Revised: 28 May 2018 / Accepted: 7 June 2018 / Published online: 6 July 2018 (c) The Author(s) 2018

\begin{abstract}
Orthogonal polynomials with respect to a weight function defined on a wedge in the plane are studied. A basis of orthogonal polynomials is explicitly constructed for two large class of weight functions and the convergence of Fourier orthogonal expansions is studied. These are used to establish analogous results for orthogonal polynomials on the boundary of the square. As an application, we study the statistics of the associated determinantal point process and use the basis to calculate Stieltjes transforms.
\end{abstract}

Keywords Orthogonal polynomials $\cdot$ Wedge $\cdot$ Boundary of square $\cdot$ Fourier orthogonal expansions

Mathematics Subject Classification $42 \mathrm{C} 05 \cdot 42 \mathrm{C} 10 \cdot 33 \mathrm{C} 50$

\section{Introduction}

Let $\Omega$ be a wedge on the plane that consists of two line segments sharing a common endpoint. For a positive measure $\mathrm{d} \mu$ defined on $\Omega$, we study orthogonal polynomials of two variables with respect to the bilinear form

$$
\langle f, g\rangle=\int_{\Omega} f(x, y) g(x, y) \mathrm{d} \mu .
$$

Communicated by Alan Edelman.

The second author was supported in part by NSF Grant DMS-1510296..

$\bowtie$ Sheehan Olver

s.olver@imperial.ac.uk

Yuan Xu

yuan@uoregon.edu

1 Department of Mathematics, Imperial College, London, UK

2 Department of Mathematics, University of Oregon, Eugene, OR 97403-1222, USA 
We also study orthogonal polynomials on the boundary of a parallelogram. Without loss of generality, we can assume that our wedge is of the form

$$
\Omega=\left\{\left(x_{1}, 1\right): x_{1} \in[0,1]\right\} \cup\left\{\left(1, x_{2}\right): x_{2} \in[0,1]\right\}
$$

and consider the bilinear form defined by

$$
\langle f, g\rangle=\int_{0}^{1} f(x, 1) g(x, 1) w_{1}(x) \mathrm{d} x+\int_{0}^{1} f(1, y) g(1, y) w_{2}(y) \mathrm{d} y .
$$

Since $\Omega$ is a subset of the zero set of a quadratic polynomial $l_{1}(x, y) l_{2}(x, y)$, where $l_{1}$ and $l_{2}$ are linear polynomials, the structure of orthogonal polynomials on $\Omega$ is very different from that of ordinary orthogonal polynomials in two variables [4] but closer to that of spherical harmonics. The latter are defined as homogeneous polynomials that satisfy the Laplace equation $\Delta Y=0$ and are orthogonal on the unit circle, which is the zero set of the quadratic polynomial $x^{2}+y^{2}-1$. The space of spherical polynomials of degree $n$ has dimension 2 for each $n \geq 1$ and, furthermore, one basis of spherical harmonics when restricted on the unit circle are $\cos n \theta$ and $\sin n \theta$, in polar coordinates $(r, \theta)$, and the Fourier orthogonal expansions in spherical harmonics coincide with the classical Fourier series.

In Sect. 2, we consider orthogonal polynomials on a wedge. The space of orthogonal polynomials of degree $n$ has dimension 2 for each $n \geq 1$, just like that of spherical harmonics, and they satisfy the equation $\partial_{1} \partial_{2} Y=0$. The main results are

- An explicit expression in terms of univariate orthogonal polynomials when $w_{1}(x)=w_{2}(x)=w(x)$ where $w$ is any weight function on $[0,1]$ (Theorem 2.2),

- Sufficient conditions for pointwise and uniform convergence (Theorem 2.4), as well as normwise convergence (Corollary 2.5),

- Explicit expression in terms of Jacobi polynomials when $w_{1}(x)=w_{\alpha, \gamma}(x)$ and $w_{2}(x)=w_{\beta, \gamma}(x)$ (Theorem 2.7),

- Sufficient conditions for normwise convergence (Theorem 2.9).

In Sect. 3 we study orthogonal polynomials on the boundary of a parallelogram, which we can assume as the square $[-1,1]^{2}$ without loss of generality. For a family of generalized Jacobi weight functions that are symmetric in both $x$ and $y$, we are able to deduce an orthogonal basis in terms of four families of orthogonal bases on the wedge in Theorem 3.2. Furthermore, the convergence of the Fourier orthogonal expansions can also be deduced in this fashion, as shown in Theorem 3.3.

In Sect. 4 we use orthogonal polynomials on the boundary of the square to construct an orthogonal basis for the weight function $w(\max \{|x|,|y|\})$ on the square $[-1,1]^{2}$. This mirrors the way in which spherical harmonics can be used to construct a basis of orthogonal polynomials for the weight function $w\left(\sqrt{x^{2}+y^{2}}\right)$ on the unit disc. However, unlike the unit disc, the orthogonal basis we constructed are no longer polynomials in $x, y$ but are polynomials of $x, y$ and $s=\max \{|x|,|y|\}$.

The study is motivated by applications. In particular, we wish to investigate how the applications of univariate orthogonal polynomials can be translated to multivariate orthogonal polynomials on curves. As a motivating example, univariate orthogonal

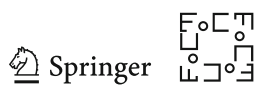


polynomials give rise to a determinantal point process that is linked to the eigenvalues of unitary ensembles of random matrix theory. In Sect. 5, we investigate the statistics of the determinantal point process generated from orthogonal polynomials on the wedge, and find experimentally that they have the same local behaviour as a Coulomb gas away from the corners/edges.

In "Appendix A", we give the Jacobi operators associated with a special case of weights on the wedge, whose entries are rational. Finally, in "Appendix B" we show that the Stieltjes transform of our family of orthogonal polynomials satisfies a recurrence that can be built out of the Jacobi operators of the orthogonal polynomials, which can in turn be used to compute Stieltjes transforms numerically. This is a preliminary step towards using these polynomials for solving singular integral equations.

\section{Orthogonal Polynomials on a Wedge}

Let $\mathcal{P}_{n}^{2}$ denote the space of homogeneous polynomials of degree $n$ in two variables; that is, $\mathcal{P}_{n}^{2}=\operatorname{span}\left\{x^{n-k} y^{k}: 0 \leq k \leq n\right\}$. Let $\Pi_{n}^{2}$ denote the space of polynomials of degree at most $n$ in two variables.

\subsection{Orthogonal Structure on a Wedge}

Given three non-collinear points, we can define a wedge by fixing one point and joining it to other points by line segments. We are interested in orthogonal polynomials on the wedge. Since the three points are non-collinear, each wedge can be mapped to

$$
\Omega=\left\{\left(x_{1}, 1\right): x_{1} \in[0,1]\right\} \cup\left\{\left(1, x_{2}\right): x_{2} \in[0,1]\right\}
$$

by an affine transform. Since the polynomial structure and the orthogonality are preserved under the affine transform, we can work with the wedge $\Omega$ without loss of generality. Henceforth we work only on $\Omega$.

Let $w_{1}$ and $w_{2}$ be two nonnegative weight functions defined on $[0,1]$. We consider the bilinear form define on $\Omega$ by

$$
\langle f, g\rangle_{w_{1}, w_{2}}:=\int_{0}^{1} f(x, 1) g(x, 1) w_{1}(x) \mathrm{d} x+\int_{0}^{1} f(1, y) g(1, y) w_{2}(y) \mathrm{d} y .
$$

Let $I$ be the polynomial ideal of $\mathbb{R}[x, y]$ generated by $(1-x)(1-y)$. If $f \in I$, then $\langle f, g\rangle_{w_{1}, w_{2}}=0$ for all $g$. The bilinear form defines an inner product on $\Pi_{n}^{2}$, modulo $I$, or equivalently, on the quotient space $\mathbb{R}[x, y] / I$.

Proposition 2.1 Let $\mathcal{H}_{n}^{2}\left(w_{1}, w_{2}\right)$ be the space of orthogonal polynomials of degree $n$ in $\mathbb{R}[x, y] / I$. Then 


$$
\operatorname{dim} \mathcal{H}_{0}^{2}\left(w_{1}, w_{2}\right)=1 \text { and } \operatorname{dim} \mathcal{H}_{n}^{2}\left(w_{1}, w_{2}\right)=2, \quad n \geq 1
$$

Furthermore, we can choose polynomials in $\mathcal{H}_{n}^{2}\left(w_{1}, w_{2}\right)$ to satisfy $\partial_{x} \partial_{y} p=0$.

Proof Since $(1-x)(1-y) \mathcal{P}_{n-2}$ is a subset of $I$, the dimension of $\operatorname{dim} \mathcal{H}_{n}^{2}\left(w_{1}, w_{2}\right) \leq 2$. Applying the Gram-Schmidt process on $\left\{1, x^{k}, y^{k}, k \geq 1\right\}$ shows that there are two orthogonal polynomials of degree exactly $n$. Both these polynomials can be written in the form of $p(x)+q(y)$, since we can use $x y \equiv x+y-1 \bmod I$ to remove all mixed terms. Evidently such polynomials satisfy $\partial_{x} \partial_{y}(p(x)+q(y))=0$.

In the next two subsections, we shall construct an orthogonal basis of $\mathcal{H}_{n}^{2}\left(w_{1}, w_{2}\right)$ for certain $w_{1}$ and $w_{2}$ and study the convergence of its Fourier orthogonal expansions. We will make use of results on orthogonal polynomials of one variable, which we briefly record here.

For $w$ defined on $[0,1]$, we let $p_{n}(w)$ denote an orthogonal polynomial of degree $n$ with respect to $w$, and let $h_{n}(w)$ denote the norm square of $p_{n}(w)$,

$$
h_{n}(w):=\int_{0}^{1}\left|p_{n}(w ; x)\right|^{2} w(x) \mathrm{d} x
$$

Let $L^{2}(w)$ denote the $L^{2}$ space with respect to $w$ on $[0,1]$. The Fourier orthogonal expansion of $f \in L^{2}(w)$ is defined by

$$
f=\sum_{n=1}^{\infty} \widehat{f_{n}}(w) p_{n}(w) \text { with } \widehat{f_{n}}(w)=\frac{1}{h_{n}(w)} \int_{0}^{1} f(y) p_{n}(w ; y) w(y) \mathrm{d} y .
$$

The Parseval identity implies that

$$
\|f\|_{L^{2}(w,[0,1])}^{2}=\sum_{n=0}^{\infty}\left|\widehat{f}_{n}(w)\right|^{2} h_{n}(w)
$$

The $n$-th partial sum of the Fourier orthogonal expansion with respect to $w$ can be written as an integral

$$
s_{n}(w ; f)(x):=\sum_{k=1}^{n} \widehat{f_{k}}(w) p_{k}(w ; x)=\int_{-1}^{1} f(y) k_{n}(w ; x, y) w(y) \mathrm{d} y,
$$

where $k_{n}(w)$ denotes the reproducing kernel defined by

$$
k_{n}(w ; x, y)=\sum_{k=0}^{n} \frac{p_{k}(w ; x) p_{k}(w ; y)}{h_{k}(w)}
$$

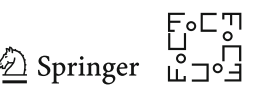




\subsection{Orthogonal Structure for $w_{1}=w_{2}$ on a Wedge}

In the case of $w_{1}=w_{2}=w$, we denote the inner product (2.1) by $\langle\cdot, \cdot\rangle_{w}$ and the space of orthogonal polynomials by $\mathcal{H}_{n}^{2}(w)$. In this case, an orthogonal basis for $\mathcal{H}_{n}^{2}(w)$ can be constructed explicitly.

Theorem 2.2 Let $w$ be a weight function on $[0,1]$ and let $\phi w(x):=(1-x)^{2} w(x)$. Define

$$
\begin{aligned}
P_{n}(x, y) & =p_{n}(w ; x)+p_{n}(w ; y)-p_{n}(w ; 1), \quad n=0,1,2, \ldots \\
Q_{n}(x, y) & =(1-x) p_{n-1}(\phi w ; x)-(1-y) p_{n-1}(\phi w ; y), \quad n=1,2, \ldots
\end{aligned}
$$

Then $\left\{P_{n}, Q_{n}\right\}$ are two polynomials in $\mathcal{H}_{n}^{2}(w)$ and they are mutually orthogonal. Furthermore,

$$
\left\langle P_{n}, P_{n}\right\rangle_{w}=2 h_{n}(w) \text { and }\left\langle Q_{n}, Q_{n}\right\rangle_{w}=2 h_{n-1}(\phi w) .
$$

Proof Since $P_{n}(x, 1)=P_{n}(1, x)$ and $Q_{n}(x, 1)=-Q_{n}(1, x)$, it follows that

$$
\left\langle P_{n}, Q_{m}\right\rangle_{w}=\int_{0}^{1} P_{n}(x, 1) Q_{m}(x, 1) w(x) \mathrm{d} x+\int_{0}^{1} P_{n}(1, x) Q_{m}(1, x) w(x) \mathrm{d} x=0
$$

for $n \geq 0$ and $m \geq 1$. Furthermore,

$$
\left\langle P_{n}, P_{m}\right\rangle_{w}=2 \int_{0}^{1} p_{n}(w ; x) p_{m}(w ; x) w(x) \mathrm{d} x=2 h_{n}(w) \delta_{n, m}
$$

by the orthogonality of $p_{n}(w)$. Similarly,

$$
\left\langle Q_{n}, Q_{m}\right\rangle_{w}=2 \int_{0}^{1} p_{n-1}(\phi w ; x) p_{m-1}(\phi w ; x)(1-x)^{2} w(x) \mathrm{d} x=2 h_{n-1}(\phi w) \delta_{n, m} .
$$

The proof is completed.

Let $L^{2}(\Omega, w)$ be the space of Lebesgue measurable functions with finite

$$
\|f\|_{L^{2}(\Omega, w)}:=\langle f, f\rangle_{w}^{\frac{1}{2}}=\left(\|f(\cdot, 1)\|_{L^{2}(w,[0,1])}^{2}+\|f(1, \cdot)\|_{L^{2}(w,[0,1])}^{2}\right)^{\frac{1}{2}}
$$

norms. For $f \in L^{2}(\Omega, w)$, its Fourier expansion is given by

$$
f=\widehat{f_{0}}+\sum_{n=1}^{\infty}\left[\widehat{f}_{P_{n}} P_{n}+\widehat{f}_{Q_{n}} Q_{n}\right]
$$


where $P_{n}$ and $Q_{n}$ are defined in Theorem 2.2 and

$$
\widehat{f_{0}}:=\frac{\langle f, 1\rangle_{w}}{\langle 1,1\rangle_{w}}, \quad \widehat{f}_{P_{n}}:=\frac{\left\langle f, P_{n}\right\rangle_{w}}{\left\langle P_{n}, P_{n}\right\rangle_{w}}, \quad \widehat{f}_{Q_{n}}:=\frac{\left\langle f, Q_{n}\right\rangle_{w}}{\left\langle Q_{n}, Q_{n}\right\rangle_{w}} .
$$

The partial sum operator $S_{n} f$ is defined by

$$
S_{n} f:=\widehat{f_{0}}+\sum_{k=1}^{n}\left[\widehat{f}_{P_{k}} P_{k}+\widehat{f}_{Q_{k}} Q_{k}\right]
$$

which can be written in terms of an integral in terms of the reproducing kernel $K_{n}(\cdot, \cdot)$,

$$
S_{n} f\left(x_{1}, x_{2}\right)=\left\langle f, K_{n}\left(\left(x_{1}, x_{2}\right), \cdot\right)\right\rangle_{w},
$$

where

$$
\begin{aligned}
K_{n}\left(\left(x_{1}, x_{2}\right),\left(y_{1}, y_{2}\right)\right):= & \frac{1}{\langle 1,1\rangle_{w}} \\
& +\sum_{k=1}^{n}\left[\frac{P_{k}\left(x_{1}, x_{2}\right) P_{k}\left(y_{1}, y_{2}\right)}{\left\langle P_{k}, P_{k}\right\rangle_{w}}+\frac{Q_{k}\left(x_{1}, x_{2}\right) Q_{k}\left(y_{1}, y_{2}\right)}{\left\langle Q_{k}, Q_{k}\right\rangle_{w}}\right] .
\end{aligned}
$$

We show that this kernel can be expressed, when restricted on $\Omega$, in terms of the reproducing kernel $k_{n}(w ; \cdot, \cdot)$ defined at $(2.3)$.

Proposition 2.3 The reproducing kernel $K_{n}(\cdot, \cdot)$ for $\langle\cdot, \cdot\rangle_{w}$ satisfies

$$
\begin{aligned}
K_{n}((x, 1),(y, 1)) & =K_{n}((1, x),(1, y)) \\
& =\frac{1}{2} k_{n}(w ; x, y)+\frac{1}{2}(1-x)(1-y) k_{n-1}(\phi w ; x, y), \\
K_{n}((x, 1),(1, y)) & =K_{n}((1, x),(y, 1)) \\
& =\frac{1}{2} k_{n}(w ; x, y)-\frac{1}{2}(1-x)(1-y) k_{n-1}(\phi w ; x, y) .
\end{aligned}
$$

Proof By (2.4) and (2.5),

$$
\begin{aligned}
K_{n}((x, 1),(y, 1))= & \frac{1}{2 h_{0}(w)}+\sum_{k=1}^{n} \frac{p_{k}(w ; x) p_{k}(w ; y)}{2 h_{k}(w)} \\
& +\sum_{k=1}^{n} \frac{(1-x) p_{k-1}(\phi w ; x)(1-y) p_{k-1}(\phi w ; y)}{2 h_{k-1}(\phi w)} \\
= & \frac{1}{2} k_{n}(w ; x, y)+\frac{1}{2}(1-x)(1-y) k_{n-1}(\phi w ; x, y) .
\end{aligned}
$$

The other case is established similar, using $Q_{k}(1, y)=-(1-y) p_{k-1}(\phi w ; y)$.

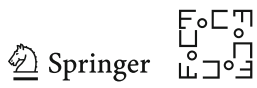


It is well-known that the kernel $k_{n}(w ; \cdot, \cdot)$ satisfies the Christoffel-Darboux formula, which plays an important role for the study of Fourier orthogonal expansion. Our formula allows us to write down an analogue of Christoffel-Darboux formula for $K_{n}(\cdot, \cdot)$, but we can derive convergence directly.

Theorem 2.4 Let $f$ be a function defined on $\Omega$. Define

$$
f_{e}(x):=\frac{1}{2}(f(x, 1)+f(1, x)) \text { and } f_{o}(x):=\frac{1}{2} \frac{f(x, 1)-f(1, x)}{1-x} .
$$

Then

$$
\begin{aligned}
& S_{n} f\left(x_{1}, 1\right)=s_{n}\left(w ; f_{e}, x_{1}\right)+\left(1-x_{1}\right) s_{n-1}\left(\phi w ; f_{o}, x_{1}\right), \\
& S_{n} f\left(1, x_{2}\right)=s_{n}\left(w ; f_{e}, x_{2}\right)-\left(1-x_{2}\right) s_{n-1}\left(\phi w ; f_{o}, x_{2}\right) .
\end{aligned}
$$

In particular, if $s_{n}\left(w ; f_{e}, x\right) \rightarrow f_{e}(x)$ and $s_{n}\left(\phi w ; f_{o}, x\right) \rightarrow f_{o}(x)$, pointwise or in the uniform norm as $n \rightarrow \infty$, then $S_{n} f(x)$ converges to $f(x)$ likewise.

Proof By our definition,

$$
\begin{aligned}
S_{n} f\left(x_{1}, 1\right)= & \int_{0}^{1} f(y, 1) K_{n}\left(\left(x_{1}, 1\right),(y, 1)\right) w(y) \mathrm{d} y \\
& +\int_{0}^{1} f(1, y) K_{n}\left(\left(x_{1}, 1\right),(1, y)\right) w(y) \mathrm{d} y \\
= & \frac{1}{2} \int_{0}^{1} f(y, 1)\left[k_{n}\left(w ; x_{1}, y\right)+\left(1-x_{1}\right)(1-y) k_{n-1}\left(\phi w ; x_{1}, y\right)\right] \\
& \times w(y) \mathrm{d} y \\
& +\frac{1}{2} \int_{0}^{1} f(1, y)\left[k_{n}\left(w ; x_{1}, y\right)-\left(1-x_{1}\right)(1-y) k_{n-1}\left(\phi w ; x_{1}, y\right)\right] \\
& \times w(y) \mathrm{d} y \\
= & s_{n}\left(w ; f_{e}, x_{1}\right)+\left(1-x_{1}\right) s_{n-1}\left(\phi w ; f_{o}, x_{1}\right) .
\end{aligned}
$$

Similarly,

$$
\begin{aligned}
& S_{n} f\left(1, x_{2}\right)=\int_{0}^{1} f(y, 1) K_{n}\left(\left(1, x_{2}\right),(y, 1)\right) w(y) \mathrm{d} y \\
& +\int_{0}^{1} f(1, y) K_{n}\left(\left(1, x_{2}\right),(1, y)\right) w(y) \mathrm{d} y \\
& =\frac{1}{2} \int_{0}^{1} f(y, 1)\left[k_{n}\left(w ; x_{2}, y\right)-\left(1-x_{2}\right)(1-y) k_{n-1}\left(\phi w ; x_{2}, y\right)\right] \\
& \times w(y) \mathrm{d} y \\
& +\frac{1}{2} \int_{0}^{1} f(1, y)\left[k_{n}\left(w ; x_{2}, y\right)+\left(1-x_{2}\right)(1-y) k_{n-1}\left(\phi w ; x_{2}, y\right)\right]
\end{aligned}
$$

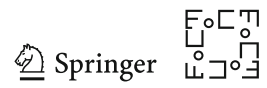




$$
\begin{aligned}
& \times w(y) \mathrm{d} y \\
= & s_{n}\left(w ; f_{e}, x_{2}\right)-\left(1-x_{2}\right) s_{n-1}\left(\phi w ; f_{o}, x_{2}\right) .
\end{aligned}
$$

Moreover, since $f_{e}(x)+(1-x) f_{o}(x)=f(x, 1)$ and $f_{e}(x)-(1-x) f_{o}(x)=f(1, x)$, it follows that

$$
\begin{aligned}
& S_{n} f\left(x_{1}, 1\right)-f\left(x_{1}\right)=s_{n}\left(w ; f_{e}, x_{1}\right)-f_{e}\left(x_{1}\right)+\left(1-x_{1}\right)\left(s_{n-1}\left(\phi w ; f_{o}, x_{1}\right)-f_{o}\left(x_{1}\right)\right), \\
& S_{n} f\left(1, x_{2}\right)-f\left(x_{2}\right)=s_{n}\left(w ; f_{e}, x_{2}\right)-f_{e}\left(x_{2}\right)-\left(1-x_{2}\right)\left(s_{n-1}\left(\phi w ; f_{o}, x_{2}\right)-f_{o}\left(x_{2}\right)\right)
\end{aligned}
$$

from which we see that the convergence of $s_{n}\left(w ; f_{e}\right)$ and $s_{n}\left(\phi w ; f_{o}\right)$ imply the convergence of $S_{n} f$.

Since $f \in L^{2}(\Omega, w)$, it is evident that $f_{e} \in L^{2}(w)$. Moreover, $f_{o} \in L^{2}(\phi w)$ since

$$
\int_{0}^{1}\left|f_{o}(x)\right|^{2} \phi w(x) \mathrm{d} x=\int_{0}^{1}|f(x, 1)-f(1, x)|^{2} w(x) \mathrm{d} x \leq 2\|f\|_{L^{2}(\Omega, w)}^{2} .
$$

In particular, $s_{n}\left(w, f_{e}\right)$ and $s_{n}\left(\phi w ; f_{o}\right)$ converge to $f_{e}$ and $f_{o}$ in $L^{2}(w)$ and in $L^{2}(\phi w)$, respectively.

Corollary 2.5 If $f \in L^{2}(\Omega, w)$, then

$$
\left\|f-S_{n}(f)\right\|_{L^{2}(\Omega, w)}^{2}=2\left(\left\|s_{n}\left(w ; f_{e}\right)-f_{e}\right\|_{L^{2}(w)}^{2}+\left\|s_{n-1}\left(\phi w ; f_{o}\right)-f_{o}\right\|_{L^{2}(\phi w)}^{2}\right) .
$$

Proof By the displayed formulas at the end of the proof of the last theorem and

$$
\int_{0}^{1}|(1-x) g(x)|^{2} w(x) \mathrm{d} x=\int_{0}^{1}|g(x)|^{2} \phi w(x) \mathrm{d} x=\|g\|_{L^{2}(\phi w)}^{2},
$$

it is easy to see that

$$
\begin{aligned}
\left\|S_{n} f-f\right\|_{L^{2}(\Omega, w)}^{2}= & \left\|s_{n}\left(w ; f_{e}\right)-f_{e}+(1-\{\cdot\})\left(s_{n-1}\left(\phi w ; f_{o}\right)-f_{o}\right)\right\|_{L^{2}(w)}^{2} \\
& +\left\|s_{n}\left(w ; f_{e}\right)-f_{e}-(1-\{\cdot\})\left(s_{n-1}\left(\phi w ; f_{o}\right)-f_{o}\right)\right\|_{L^{2}(w)}^{2} \\
= & 2\left(\left\|s_{n}\left(w ; f_{e}\right)-f_{e}\right\|_{L^{2}(w)}^{2}+\left\|s_{n-1}\left(\phi w ; f_{o}\right)-f_{o}\right\|_{L^{2}(\phi w)}^{2}\right),
\end{aligned}
$$

where we have used the identity $(a+b)^{2}+(a-b)^{2}=2\left(a^{2}+b^{2}\right)$.

\subsection{Orthogonal Structure on a Wedge with Jacobi Weight Functions}

For $\alpha, \gamma>-1$, let $w_{\alpha, \gamma}$ be the Jacobi weight function defined by

$$
w_{\alpha, \gamma}(x):=x^{\alpha}(1-x)^{\gamma}, \quad x \in[0,1]
$$

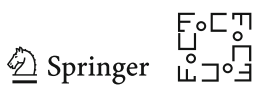


We consider the inner product $\langle\cdot, \cdot\rangle_{w_{1}, w_{2}}$ defined in (2.1) with $w_{1}(x)=w_{\alpha, \gamma}(x)$ and $w_{2}(x)=w_{\beta, \gamma}(x)$. More specifically, for $\alpha, \beta, \gamma>-1$ and $\sigma>0$, we define

$$
\begin{aligned}
\langle f, g\rangle_{\alpha, \beta, \gamma}:= & c_{\alpha, \gamma} \int_{0}^{1} f(x, 1) g(x, 1) w_{\alpha, \gamma}(x) \mathrm{d} x \\
& +\sigma c_{\beta, \gamma} \int_{0}^{1} f(1, y) g(1, y) w_{\beta, \gamma}(y) \mathrm{d} y,
\end{aligned}
$$

where

$$
c_{\alpha, \gamma}:=\left(\int_{0}^{1} w_{\alpha, \gamma}(x) \mathrm{d} x\right)^{-1}=\frac{\Gamma(\gamma+\alpha+2)}{\Gamma(\gamma+1) \Gamma(\alpha+1)} .
$$

\subsubsection{Orthogonal Structure}

We need to construct an explicit basis of $\mathcal{H}_{n}^{2}\left(w_{\alpha, \gamma}, w_{\beta, \gamma}\right)$. The case $\alpha=\beta$ can be regarded as a special case of Theorem 2.2. The case $\alpha \neq \beta$ is much more complicated, for which we need several properties of the Jacobi polynomials.

Let $P_{n}^{(\alpha, \beta)}$ denote the usual Jacobi polynomial of degree $n$ defined on $[-1,1]$. Then $P_{n}^{(\gamma, \alpha)}(2 x-1)$ is an orthogonal polynomial with respect to $w_{\alpha, \gamma}$ on $[0,1]$. Moreover,

$$
\begin{aligned}
h_{n}^{\alpha, \gamma}: & =c_{\alpha, \gamma} \int_{0}^{1}\left[P_{n}^{(\gamma, \alpha)}(2 x-1)\right]^{2} w_{\alpha, \gamma}(x) \mathrm{d} x \\
& =\frac{(\gamma+1)_{n}(\alpha+1)_{n}(n+\gamma+\alpha+1)}{n !(\gamma+\alpha+2)_{n}(2 n+\gamma+\alpha+1)}
\end{aligned}
$$

by [12, (4.3.3)]. Furthermore, $P_{n}^{(\alpha, \beta)}(1)=\left(\begin{array}{c}n+\alpha \\ n\end{array}\right)$ and, in particular, $P_{n}^{(0, \beta)}(1)=1$. Our construction relies on the following lemma.

Lemma 2.6 For $m>n \geq 0$,

$$
\begin{aligned}
I_{m, n}^{\alpha, \gamma}: & =c_{\alpha, \gamma} \int_{0}^{1} P_{n}^{(\gamma, \alpha)}(2 x-1) P_{m-1}^{(\gamma+2, \alpha)}(2 x-1)(1-x)^{\gamma+1} x^{\alpha} \mathrm{d} x \\
& = \begin{cases}0, & n>m, \\
\frac{-m(\gamma+1)_{m}(\alpha+1)_{m}}{m !(2 m+\gamma+\alpha+1)(\gamma+\alpha+2)_{m}}, & n=m, \\
\frac{(\gamma+1)(\alpha+1)_{m-1}(\gamma+1)_{n}}{(\gamma+\alpha+2)_{m} n !}, & n<m .\end{cases}
\end{aligned}
$$

Proof Since $P_{n}^{(\gamma, \alpha)}(2 x-1)$ is an orthogonal polynomial of degree $n$ with respect to $(1-x)^{\gamma} x^{\alpha}$ on $[0,1], I_{m, n}^{\gamma, \alpha}=0$ for $n>m$ holds trivially. For $m \geq n$, we need two identities of Jacobi polynomials. The first one is, see [12, (4.5.4)] or [9, (18.9.6)], 


$$
\begin{aligned}
& (2 m+\gamma+\alpha+1)(1-x) P_{m-1}^{(\gamma+2, \alpha)}(2 x-1) \\
& \quad=(m+\gamma+1) P_{m-1}^{(\gamma+1, \alpha)}(2 x-1)-m P_{m}^{(\gamma+1, \alpha)}(2 x-1)
\end{aligned}
$$

and the second one is the expansion, see [9, (18.18.14)],

$$
\begin{aligned}
P_{m}^{(\gamma+1, \alpha)}(2 x-1)= & \frac{(\alpha+1)_{m}}{(\gamma+\alpha+2)_{m}} \sum_{k=0}^{m} \frac{(\gamma+\alpha+1)_{k}(2 k+\gamma+\alpha+1)}{(\alpha+1)_{k}(\gamma+\alpha+1)} \\
& \times P_{k}^{(\gamma, \alpha)}(2 x-1) .
\end{aligned}
$$

Putting them together shows that

$$
\begin{aligned}
(1-x) P_{m-1}^{(\gamma+2, \alpha)}(2 x-1)= & \frac{(\gamma+1)(\alpha+1)_{m-1}}{(\gamma+\alpha+1)_{m+1}} \\
& \times \sum_{k=0}^{m-1} \frac{(\gamma+\alpha+1)_{k}(2 k+\gamma+\alpha+1)}{(\alpha+1)_{k}} P_{k}^{(\gamma, \alpha)}(2 x-1) \\
& -\frac{m}{m+\gamma+\alpha+1} P_{m}^{(\gamma, \alpha)}(2 x-1) .
\end{aligned}
$$

Substituting this expression into $I_{m, n}^{\gamma, \alpha}$ and using the orthogonality of the Jacobi polynomials and (2.10), we conclude that, for $m-1 \geq n$,

$$
I_{m, n}^{\alpha, \gamma}=\frac{(\gamma+1)(\alpha+1)_{m-1}}{(\gamma+\alpha+2)_{m}} \frac{(\gamma+1)_{n}}{n !} .
$$

Hence, the case $m>n$ follows. The same argument works for the case $n=m$.

What is of interest for us is the fact that the dependence of $I_{m, n}^{\gamma, \alpha}$ on $n$ and $\alpha$ is separated, which is critical to prove that $Q_{n}$ in the next theorem is orthogonal.

Theorem 2.7 Let $P_{0}(x, y)=1$ and, for $n=1,2, \ldots$, define

$$
\begin{aligned}
P_{n}(x, y)= & P_{n}^{(\gamma, \alpha)}(2 x-1)+P_{n}^{(\gamma, \beta)}(2 y-1)-\left(\begin{array}{c}
n+\gamma \\
n
\end{array}\right), \\
Q_{n}(x, y)= & \frac{(\gamma+\alpha+2)_{n}}{(\alpha+1)_{n-1}}(1-x) P_{n-1}^{(\gamma+2, \alpha)}(2 x-1) \\
& -\sigma^{-1} \frac{(\gamma+\beta+2)_{n}}{(\beta+1)_{n-1}}(1-y) P_{n-1}^{(\gamma+2, \beta)}(2 y-1) .
\end{aligned}
$$

Then $\left\{P_{n}, Q_{n}\right\}$ are two polynomials in $\mathcal{H}_{n}^{2}\left(w_{\alpha, \gamma}, w_{\beta, \gamma}\right)$ and

$$
\left\langle P_{n}, Q_{n}\right\rangle_{\alpha, \beta, \gamma}=\frac{(\beta-\alpha)(\gamma+1)_{n+1}}{(2 n+\gamma+\alpha+1)(2 n+\gamma+\beta+1)(n-1) !} .
$$

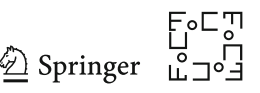


In particular, the two polynomials are orthogonal to each other if $\beta=\alpha$. Furthermore

$$
\begin{aligned}
\left\langle P_{n}, P_{n}\right\rangle_{\alpha, \beta, \gamma}= & h_{n}^{\alpha, \gamma}+\sigma h_{n}^{\beta, \gamma} \\
\left\langle Q_{n}, Q_{n}\right\rangle_{\alpha, \beta, \gamma}= & \frac{(\gamma+1)_{2}(\alpha+\gamma+2)_{n}^{2}}{(\alpha+\gamma+2)_{2}(\alpha+1)_{n-1}^{2}} h_{n-1}^{\alpha, \gamma+2} \\
& +\sigma^{-1} \frac{(\gamma+1)_{2}(\beta+\gamma+2)_{n}^{2}}{(\beta+\gamma+2)_{2}(\beta+1)_{n-1}^{2}} h_{n-1}^{\beta, \gamma+2} .
\end{aligned}
$$

Proof Since $P_{n}^{(\gamma, \alpha)}(1)=P_{n}^{(\gamma, \beta)}(1)=\left(\begin{array}{c}n+\gamma \\ n\end{array}\right)$, our definition shows that

$$
\left\langle P_{n}, Q_{m}\right\rangle_{\alpha, \beta, \gamma}=\frac{(\gamma+\alpha+2)_{m}}{(\alpha+1)_{m-1}} I_{m, n}^{\alpha, \gamma}-\frac{(\gamma+\beta+2)_{m}}{(\beta+1)_{m-1}} I_{m, n}^{\beta, \gamma} .
$$

By the identity in the previous lemma, if $n>m$, then $\left\langle P_{n}, Q_{m}\right\rangle_{\alpha, \beta, \gamma}=0$ since both $I_{m, n}^{\alpha, \gamma}=0$ and $I_{m, n}^{\beta, \gamma}=0$, whereas if $n<m$, then

$$
\left\langle P_{n}, Q_{m}\right\rangle_{\alpha, \beta, \gamma}=\frac{(\gamma+1)(\gamma+1)_{n}}{n !}-\frac{(\gamma+1)(\gamma+1)_{n}}{n !}=0 .
$$

The case $n=m$ follows from a simple calculation. Moreover, for $m \neq n$,

$$
\begin{aligned}
\left\langle P_{n}, P_{m}\right\rangle_{\alpha, \beta, \gamma}= & c_{\alpha, \gamma} \int_{0}^{1} P_{n}^{(\gamma, \alpha)}(2 x-1) P_{m}^{(\gamma, \alpha)}(2 x-1)(1-x)^{\gamma} x^{\alpha} \mathrm{d} x \\
& +c_{\gamma, \gamma} \int_{0}^{1} P_{n}^{(\beta, \gamma)}(2 x-1) P_{m}^{(\gamma, \beta)}(2 x-1)(1-x)^{\gamma} x^{\beta} \mathrm{d} x=0
\end{aligned}
$$

by the orthogonality of the Jacobi polynomials, and it is equal to $h_{n}^{\gamma, \alpha}+h_{n}^{\gamma, \beta}$ for $m=n$. Similarly,

$$
\begin{gathered}
\left\langle Q_{n}, Q_{m}\right\rangle_{\alpha, \beta, \gamma}=\frac{(\gamma+\alpha+2)_{m}}{(\alpha+1)_{m-1}} c_{\alpha, \gamma} \int_{0}^{1} P_{n-1}^{(\gamma+2, \alpha)}(2 x-1) P_{m-1}^{(\gamma+2, \alpha)}(2 x-1) \\
(1-x)^{\gamma+2} x^{\alpha} \mathrm{d} x \\
+\sigma^{-1} \frac{(\gamma+\beta+2)_{m}}{(\beta+1)_{m-1}} c_{\beta, \gamma} \int_{0}^{1} P_{n-1}^{(\gamma+2, \beta)}(2 x-1) P_{m-1}^{(\gamma+2, \beta)}(2 x-1)(1-x)^{\gamma+2} x^{\beta} \mathrm{d} x \\
=0 .
\end{gathered}
$$

To derive the norm of $\left\langle Q_{n}, Q_{n}\right\rangle$, we need to use $c_{\gamma, \alpha}=(\gamma+1)_{2} /(\alpha+\gamma+2)_{2} c_{\gamma+2, \alpha}$. The proof is completed.

Corollary 2.8 For $n=1,2, \ldots$, define

$$
R_{n}(x, y)=Q_{n}(x, y)-\frac{\left\langle P_{n}, Q_{n}\right\rangle_{\alpha, \beta, \gamma}}{h_{n}^{\gamma, \alpha}+\sigma h_{n}^{\gamma, \beta}} P_{n}(x, y) .
$$

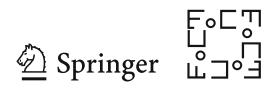


Then, for $\alpha \neq \beta,\left\{P_{n}, R_{n}\right\}$ are two polynomials in $\mathcal{H}_{n}^{2}\left(w_{\alpha, \beta, \gamma}\right)$ and they are mutually orthogonal. Moreover,

$$
\left\langle R_{n}, R_{n}\right\rangle_{\alpha, \beta, \gamma}=\left\langle Q_{n}, Q_{n}\right\rangle_{\alpha, \beta, \gamma}-\frac{\left\langle P_{n}, Q_{n}\right\rangle_{\alpha, \beta, \gamma}}{\left\langle P_{n}, P_{n}\right\rangle_{\alpha, \beta, \gamma}}
$$

\subsubsection{Fourier Orthogonal Expansions}

Let $L^{2}\left(\Omega, w_{\alpha, \gamma}, w_{\beta, \gamma}\right)$ be the space of functions defined on $\Omega$ such that $f(1,1)$ is finite and the norm

$$
\begin{aligned}
\|f\|_{L^{2}\left(\Omega, w_{\alpha, \gamma}, w_{\beta, \gamma}\right)}= & \left(c_{\alpha, \gamma} \int_{0}^{1}|f(x, 1)|^{2} w_{\alpha, \gamma}(x) \mathrm{d} x\right. \\
& \left.+\sigma c_{\beta, \gamma} \int_{0}^{1}|f(1, y)|^{2} w_{\beta, \gamma}(y) \mathrm{d} y\right)^{\frac{1}{2}}
\end{aligned}
$$

is finite for every $f$ in this space. For $f \in L^{2}\left(\Omega, w_{\alpha, \gamma}, w_{\beta, \gamma}\right)$ we consider the Fourier orthogonal expansion with respect to $\langle\cdot, \cdot\rangle_{\alpha, \beta, \gamma}$. With respect to the orthogonal basis $\left\{P_{n}, R_{n}\right\}$ in Theorem 2.7 and Corollary 2.8, the Fourier orthogonal expansion is defined by

$$
f=\widehat{f_{0}}+\sum_{n=1}^{\infty}\left[\widehat{f}_{P_{n}} P_{n}+\widehat{f}_{R_{n}} R_{n}\right]
$$

where

$$
\widehat{f_{0}}:=\frac{\langle f, 1\rangle_{\alpha, \beta, \gamma}}{\langle 1,1\rangle_{\alpha, \beta, \gamma}}, \quad \widehat{f}_{P_{n}}:=\frac{\left\langle f, P_{n}\right\rangle_{\alpha, \beta, \gamma}}{\left\langle P_{n}, P_{n}\right\rangle_{\alpha, \beta, \gamma}}, \quad \widehat{f}_{R_{n}}:=\frac{\left\langle f, R_{n}\right\rangle_{\alpha, \beta, \gamma}}{\left\langle R_{n}, R_{n}\right\rangle_{\alpha, \beta, \gamma}} .
$$

Its $n$-th partial sum is defined by

$$
S_{n}^{\alpha, \beta, \gamma} f:=\widehat{f_{0}}+\sum_{k=1}^{n}\left[\widehat{f}_{P_{k}} P_{k}+\widehat{f}_{R_{k}} R_{k}\right]
$$

In this case, we do not have a closed form for the reproducing kernel with respect to $\langle\cdot, \cdot\rangle_{\alpha, \beta, \gamma}$. Nevertheless, we can relate the convergence of the Fourier orthogonal expansions to that of the Fourier-Jacobi series. For $w_{\alpha, \gamma}$, we denote the partial sum defined in (2.2) by $s_{n}^{\alpha, \gamma} f$.

For $f$ defined on $\Omega$, we define $f_{1}(x)=f(x, 1)$ and $f_{2}(x)=f(1, x)$, and

$$
g_{1}(x):=\frac{f(x, 1)-f(1,1)}{1-x} \text { and } g_{2}(y):=\frac{f(1, y)-f(1,1)}{1-y} .
$$

It is easy to see that if $f(\cdot, 1) \in L^{2}\left(w_{\alpha, \gamma},[0,1]\right)$, then $g_{1} \in L^{2}\left(w_{\alpha, \gamma+2},[0,1]\right)$, and if $f(1, \cdot) \in L^{2}\left(w_{\beta, \gamma},[0,1]\right)$, then $g_{2} \in L^{2}\left(w_{\beta, \gamma+2},[0,1]\right)$.

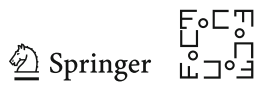


Theorem 2.9 Let $\alpha, \beta, \gamma>-1$. Then the Fourier orthogonal expansion converges in $f \in L^{2}\left(\Omega, w_{\alpha, \gamma}, w_{\beta, \gamma}\right)$. Furthermore, for $f(\cdot, 1) \in L^{2}\left(w_{\alpha, \gamma}\right)$ and $f(1, \cdot) \in$ $L^{2}\left(w_{\beta, \gamma}\right)$,

$$
\begin{aligned}
\left\|f-S_{n}^{\alpha, \beta, \gamma} f\right\|_{\alpha, \beta, \gamma} \leq & c\left(\left\|f_{1}-s_{n}^{\alpha, \gamma} f_{1}\right\|_{L^{2}\left(w_{\alpha, \gamma}\right)}+\left\|f_{2}-s_{n}^{\beta, \gamma} f_{2}\right\|_{L^{2}\left(w_{\beta, \gamma}\right)}\right) \\
& +c\left(\left\|g_{1}-s_{n}^{\alpha, \gamma+2} g_{1}\right\|_{L^{2}\left(w_{\alpha, \gamma+2}\right)}+\left\|g_{2}-s_{n}^{\beta, \gamma+2} g_{2}\right\|_{L^{2}\left(w_{\beta, \gamma+2}\right)}\right),
\end{aligned}
$$

where $c$ is a constant that depends only on $\alpha, \beta, \gamma$.

Proof Since polynomials are dense on $\Omega$, by the Weierstrass theorem, the orthogonal basis $\left\{P_{n}, R_{n}\right\}$ is complete, so that the Fourier orthogonal expansion converges in $L^{2}\left(\Omega, w_{\alpha, \gamma}, w_{\beta, \gamma}\right)$. By the Parseval identity,

$$
\left\|f-S_{n}^{\alpha, \beta, \gamma} f\right\|_{\alpha, \beta, \gamma}^{2}=\sum_{k=n+1}^{\infty}\left|\widehat{f}_{P_{k}}\right|^{2}\left\langle P_{k}, P_{k}\right\rangle_{\alpha, \beta, \gamma}+\sum_{k=n+1}^{\infty}\left|\widehat{f}_{R_{k}}\right|^{2}\left\langle R_{k}, R_{k}\right\rangle_{\alpha, \beta, \gamma} .
$$

Throughout this proof we use the convention $A \sim B$ if $c_{1} B \leq A \leq c_{2} A$, where $c_{1}$ and $c_{2}$ are constants that are independent of varying parameters in $A$ and $B$. By (2.10) and the fact that $\Gamma(n+\alpha+1) / n ! \sim n^{\alpha}$, it is easy to see that $h_{n}^{\alpha, \gamma} \sim n^{-1}$, so that

$$
\left\langle P_{n}, P_{n}\right\rangle_{\alpha, \beta, \gamma} \sim n^{-1}, \quad\left\langle Q_{n}, Q_{n}\right\rangle_{\alpha, \beta, \gamma} \sim n^{2 \gamma+3}, \quad\left\langle P_{n}, Q_{n}\right\rangle_{\alpha, \beta, \gamma} \sim n^{\gamma}
$$

and, consequently,

$$
\left\langle R_{n} R_{n}\right\rangle_{\alpha, \beta, \gamma} \sim n^{2 \gamma+3}-n^{2 \gamma} / n^{-1} \sim n^{2 \gamma+3}
$$

The Fourier-Jacobi coefficients of $f_{1}$ and $f_{2}$ are denoted by $\widehat{f}_{1_{n}}^{\alpha, \gamma}$ and $\widehat{f}_{2}{ }_{n}^{\beta, \gamma}$, respectively. It follows readily that $\widehat{f}_{P_{n}} \sim \widehat{f}_{1_{n}}^{\alpha, \gamma}+\widehat{f}_{2}^{\beta, \gamma}$, consequently,

$$
\begin{aligned}
\sum_{k=n+1}^{\infty}\left|\widehat{f}_{P_{k}}\right|^{2}\left\langle P_{k}, P_{k}\right\rangle_{\alpha, \beta, \gamma} & \leq c \sum_{k=n+1}^{\infty}\left(\left|\widehat{f}_{1 k}^{\alpha, \gamma}\right|^{2} h_{k}^{\alpha, \gamma}+\left|\widehat{f}_{2 k}^{\beta, \gamma}\right|^{2} h_{k}^{\beta, \gamma}\right) \\
& \leq c\left(\left\|f_{1}-s_{n}^{\alpha, \gamma} f_{1}\right\|_{L^{2}\left(w_{\alpha, \gamma}\right)}+\left\|f_{2}-s_{n}^{\beta, \gamma} f_{2}\right\|_{L^{2}\left(w_{\beta, \gamma}\right)}\right)
\end{aligned}
$$

We now consider the estimate for $R_{n}$ part. By the definition of $R_{n}$,

$$
\left\langle f, R_{n}\right\rangle_{\alpha, \beta, \gamma} \sim\left\langle f, Q_{n}\right\rangle_{\alpha, \beta, \gamma}-n^{\gamma+1}\left\langle f, P_{n}\right\rangle_{\alpha, \beta, \gamma}
$$

It is easy to see that 


$$
\begin{aligned}
& \sum_{k=n+1}^{\infty} \frac{\left|k^{\gamma+1}\left\langle f, P_{k}\right\rangle_{\alpha, \beta, \gamma}\right|^{2}}{\left\langle R_{k}, R_{k}\right\rangle_{\alpha, \beta, \gamma}} \sim \sum_{k=n+1}^{\infty} k^{-1}\left|\left\langle f, P_{k}\right\rangle_{\alpha, \beta, \gamma}\right|^{2} \\
& \sim \sum_{k=n+1}^{\infty} k^{-2}\left|\widehat{f}_{P_{k}}\right|^{2}\left\langle P_{k}, P_{k}\right\rangle_{\alpha, \beta, \gamma}
\end{aligned}
$$

so that we only have to work with the term $\left\langle f, Q_{k}\right\rangle_{\alpha, \beta, \gamma}$. The definition of $Q_{k}$ shows that $\left\langle 1, Q_{k}\right\rangle_{\alpha, \beta, \gamma}=0$, which leads to the identity

$$
\begin{aligned}
\left\langle f, Q_{k}\right\rangle_{\alpha, \beta, \gamma}= & \frac{(\gamma+\alpha+2)_{k}}{(\alpha+k)_{k-1}} c_{\alpha, \gamma} \int_{0}^{1}(f(x, 1)-f(1,1)) Q_{k}(x, 1) x^{\alpha}(1-x)^{\gamma} \mathrm{d} x \\
& +\frac{(\gamma+\beta+2)_{k}}{(\beta+n)_{k-1}} c_{\beta, \gamma} \int_{0}^{1}(f(1, y)-f(1,1)) Q_{k}(1, y) y^{\beta}(1-y)^{\gamma} \mathrm{d} y \\
= & \frac{(\gamma+\alpha+2)_{k}}{(\alpha+k)_{k-1}} \widehat{g}_{1}^{\alpha, \gamma+2} h_{k}^{\alpha, \gamma+2}+\frac{(\gamma+\beta+2)_{k}}{(\beta+n)_{k-1}} \widehat{g}_{2}^{\beta, \gamma+2} h_{k}^{\beta, \gamma+2} .
\end{aligned}
$$

Consequently, it follows that

$$
\begin{aligned}
& \sum_{k=n+1}^{\infty} \frac{\left|\left\langle f, Q_{k}\right\rangle_{\alpha, \beta, \gamma}\right|^{2}}{\left\langle R_{k}, R_{k}\right\rangle_{\alpha, \beta, \gamma}} \\
& \quad \leq c \sum_{k=n+1}^{\infty}\left(k\left|\widehat{g}_{1}^{\alpha, \gamma+2} h_{k}^{\alpha, \gamma+2}\right|^{2}+k\left|\widehat{g}_{2}^{\beta, \gamma+2} h_{k}^{\beta, \gamma+2}\right|^{2}\right) \\
& \quad \leq c \sum_{k=n+1}^{\infty}\left(\left|\widehat{g}_{1}^{\alpha, \gamma+2}\right|^{2} h_{k}^{\alpha, \gamma+2}+\left|\widehat{g}_{2}^{\beta, \gamma+2}\right|^{2} h_{k}^{\beta, \gamma+2}\right) \\
& \quad=c\left(\left\|g_{1}-s_{n}^{\alpha, \gamma+2} g_{1}\right\|_{L^{2}\left(w_{\alpha, \gamma+2}\right)}+\left\|g_{2}-s_{n}^{\beta, \gamma+2} g_{2}\right\|_{L^{2}\left(w_{\beta, \gamma+2}\right)}\right) .
\end{aligned}
$$

The proof is completed.

\section{Orthogonal Polynomials on the Boundary of the Square}

Using the results in the previous section, we can study orthogonal polynomials on a parallelogram. Since orthogonal structure is preserved under an affine transformation, we can assume without loss of generality that the parallelogram is the square $[-1,1]^{2}$.

For $\alpha, \gamma>-1$, let $\varpi_{\alpha, \gamma}$ be the weight function

$$
\varpi_{\alpha, \gamma}(x):=|x|^{2 \alpha+1}\left(1-x^{2}\right)^{\gamma} .
$$

We consider orthogonal polynomials of two variables on the boundary of $[-1,1]^{2}$ with respect to the bilinear form

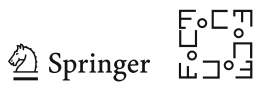




$$
\begin{aligned}
\langle f, g\rangle= & c_{\alpha, \gamma} \int_{-1}^{1}[f(x,-1) g(x,-1)+f(x, 1) g(x, 1)] \varpi_{\alpha, \gamma}(x) \mathrm{d} x \\
& +c_{\beta, \gamma} \int_{-1}^{1}[f(-1, y) g(-1, y)+f(1, y) g(1, y)] \varpi_{\beta, \gamma}(y) \mathrm{d} y
\end{aligned}
$$

for $\alpha, \beta, \gamma>-1$. Since $\left(1-x^{2}\right)\left(1-y^{2}\right)$ vanishes on the boundary of the square, the bilinear form defines an inner product modulo the ideal generated by this polynomial, or in the space

$$
\mathbb{R}[x, y] / I:=\mathbb{R}[x, y] /\left\langle\left(1-x^{2}\right)\left(1-y^{2}\right)\right\rangle .
$$

Let $\mathcal{B} \mathcal{V}_{n}^{2}$ denote the space of orthogonal polynomials in $\mathbb{R}[x, y] / I$ with respect to the inner product $\langle\cdot, \cdot\rangle$.

Proposition 3.1 For $n \geq 0$, the dimension of $\mathcal{B} \mathcal{V}_{n}^{2}$ is given by

$$
\operatorname{dim} \mathcal{B} \mathcal{V}_{n}^{2}=n+1, \quad n=0,1,2, \quad \text { and } \quad \operatorname{dim} \mathcal{B} \mathcal{V}_{n}^{2}=4, \quad n \geq 3
$$

Recall that the inner product $\langle\cdot, \cdot\rangle_{\alpha, \beta, \gamma}$ studied in the previous section contains a fixed parameter $\sigma$. For fixed $\alpha, \beta$ and $\delta_{1}, \delta_{2} \in\{0,1\}$, we define $p_{m, 1}^{\alpha+\delta_{1}, \beta+\delta_{2}, \gamma}$ and $p_{m, 2}^{\alpha+\delta_{1}, \beta+\delta_{2}, \gamma}$ to be a basis of $\mathcal{H}_{m}^{2}\left(w_{\alpha+\delta_{1}, \gamma}, w_{\beta+\delta_{2}, \gamma}\right)$ for a particular choice of $\sigma$ defined by

$$
\sigma_{\delta_{1}, \delta_{2}}=\frac{c_{\beta, \gamma} c_{\alpha+\delta_{1}, \gamma}}{c_{\alpha, \gamma} c_{\beta+\delta_{2}, \gamma}} .
$$

For example, $p_{m, i}^{\alpha, \beta, \gamma}$ are defined with $\sigma_{0,0}=1$ and $p_{m, i}^{\alpha+1, \beta, \gamma}$ are defined with $\sigma_{1,1}=$ $(\alpha+\gamma+2) /(\alpha+1)$. For each pair of $\alpha+\delta_{1}, \beta+\delta_{2}$, we can choose, for example, $p_{m, 1}^{\alpha+\delta_{1}, \beta+\delta_{2}, \gamma}=P_{m}$ defined in (2.12) and take $p_{m, 2}^{\alpha+\delta_{1}, \beta+\delta_{2}, \gamma}=Q_{m}$ defined in (2.13) or $p_{m, 2}^{\alpha+1, \beta+1, \gamma}=R_{m}$ defined in (2.15).

Theorem 3.2 For $n=0,1,2$, a basis for $\mathcal{B} \mathcal{V}_{n}$ is denoted by $Y_{n, i}$ and given by

$$
\begin{aligned}
& Y_{0,1}(x, y)=1, \quad Y_{1,1}(x, y)=x \quad Y_{1,2}(x, y)=y, \\
& Y_{2,1}(x, y)=p_{1,1}^{\alpha, \beta, \gamma}\left(x^{2}, y^{2}\right), \quad Y_{2,2}(x, y)=x y, \quad Y_{2,3}(x, y)=p_{1,2}^{\alpha, \beta, \gamma}\left(x^{2}, y^{2}\right) .
\end{aligned}
$$

For $n \geq 3$, the four polynomials in $\mathcal{B V}_{n}^{2}$ that are linearly independent modulo the ideal can be given by

$$
\begin{aligned}
& Y_{2 m, 1}(x, y)=p_{m, 1}^{\alpha, \beta, \gamma}\left(x^{2}, y^{2}\right), \\
& Y_{2 m, 2}(x, y)=p_{m, 2}^{\alpha, \beta, \gamma}\left(x^{2}, y^{2}\right), \\
& Y_{2 m, 3}(x, y)=x y p_{m-1,1}^{\alpha+1, \beta+1, \gamma}\left(x^{2}, y^{2}\right), \\
& Y_{2 m, 4}(x, y)=x y p_{m-1,2}^{\alpha+1, \beta+1, \gamma}\left(x^{2}, y^{2}\right)
\end{aligned}
$$


for $n=2 m \geq 2$, and

$$
\begin{aligned}
& Y_{2 m+1,1}(x, y)=x p_{m, 1}^{\alpha+1, \beta, \gamma}\left(x^{2}, y^{2}\right), \\
& Y_{2 m+1,2}(x, y)=x p_{m, 2}^{\alpha+1, \beta, \gamma}\left(x^{2}, y^{2}\right), \\
& Y_{2 m+1,3}(x, y)=y p_{m, 1}^{\alpha, \beta+1, \gamma}\left(x^{2}, y^{2}\right), \\
& Y_{2 m+1,4}(x, y)=y p_{m, 2}^{\alpha, \beta+1, \gamma}\left(x^{2}, y^{2}\right)
\end{aligned}
$$

for $n=2 m+1 \geq 3$. In particular, these bases satisfy the equation $\partial_{x}^{2} \partial_{y}^{2} u=0$.

Proof The proof relies on the parity of the integrals. For example, it is easy to see that $\left\langle x f\left(x^{2}, y^{2}\right), g\left(x^{2}, y^{2}\right)\right\rangle=0$ and $\left\langle y f\left(x^{2}, y^{2}\right), g\left(x^{2}, y^{2}\right)\right\rangle=0$ for any polynomials $f$ and $g$, which implies, in particular, that $\left\langle Y_{2 m, i}, Y_{2 n+1, j}\right\rangle=0$ for $i, j=1,2,3,4$. Furthermore, it is easy to see that $\left\langle x y f\left(x^{2}, y^{2}\right), g\left(x^{2}, y^{2}\right)\right\rangle=0$ for any polynomials $f$ and $g$. Hence, $\left\langle Y_{2 m, i}, Y_{2 k, j}\right\rangle=0$ for $i=1,2$ and $j=3$, 4. Furthermore, using the relation

$$
\int_{-1}^{1} f\left(x^{2}\right)|x|^{2 \alpha+1}\left(1-x^{2}\right)^{\gamma} \mathrm{d} x=\int_{0}^{1} f(x) x^{\alpha}(1-x)^{\gamma} \mathrm{d} x,
$$

it is easy to see that

$$
\begin{aligned}
& \left\langle Y_{2 m, i}, Y_{2 k, j}\right\rangle=\left\langle p_{m, i}^{\alpha, \beta, \gamma}, p_{k, j}^{\alpha, \beta, \gamma}\right\rangle_{\alpha, \beta, \gamma}, \quad i, j=1,2 \\
& \left\langle Y_{2 m, i}, Y_{2 k, j}\right\rangle=\frac{c_{\alpha, \gamma}}{c_{\alpha+1, \gamma}}\left\langle p_{m, i}^{\alpha+1, \beta+1, \gamma}, p_{k, j}^{\alpha+1, \beta+1, \gamma}\right\rangle_{\alpha+1, \beta+1, \gamma}, \quad i, j=3,4,
\end{aligned}
$$

where in the second identity, we have adjusted the normalization constants of integrals from $c_{\alpha, \gamma}$ and $c_{\beta, \gamma}$ to $c_{\alpha+1, \gamma}$ and $c_{\beta+1, \gamma}$, respectively, and used our choice of $\sigma_{1,1}$. Hence, with our choice of $\sigma_{0,0}$ and $\sigma_{1,1}$, we see that $Y_{2 m, i}$ is orthogonal to $Y_{2 k, j}$ for $i, j=1,2$ and $i, j=3,4$, respectively. Similarly, by the same consideration, we obtain that

$$
\begin{aligned}
& \left\langle Y_{2 m+1, i}, Y_{2 k+1, j}\right\rangle=\frac{c_{\alpha, \gamma}}{c_{\alpha+1, \gamma}}\left\langle p_{m, i}^{\alpha+1, \beta, \gamma}, p_{k, j}^{\alpha+1, \beta, \gamma}\right\rangle_{\alpha+1, \beta, \gamma}, \quad i, j=1,2 \\
& \left\langle Y_{2 m+1, i}, Y_{2 k+1, j}\right\rangle=\left\langle p_{m, i}^{\alpha, \beta+1, \gamma}, p_{k, j}^{\alpha, \beta+1, \gamma}\right\rangle_{\alpha, \beta+1, \gamma}, \quad i, j=3,4
\end{aligned}
$$

which shows, with our choice of $\sigma_{0,1}$ and $\sigma_{1,0}$, that $Y_{2 m+1, i}$ is orthogonal to $Y_{2 k+1, j}$ for $i, j=1,2$ and $i, j=3,4$, respectively. Finally, since $\partial_{x} \partial_{y} p_{n, i}^{\alpha, \beta}(x, y)=0$, we see that $Y_{n, j}=\xi(x, y) u(x)+\eta(x, y) v(x)$, where $\xi$ and $\eta$ are linear polynomial of $x, y$, so that it is evident that $\partial_{x}^{2} \partial_{y}^{2} Y_{n, j}(x, y)=0$.

In our notation, the case $\alpha=-\frac{1}{2} \beta=-\frac{1}{2}$ and $\gamma=0$ corresponds to the inner product in which the integrals are unweighted.

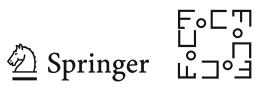


Let $L^{2}\left([-1,1]^{2}, \varpi_{\alpha, \gamma}, \varpi_{\beta, \gamma}\right)$ be the space of functions defined on the boundary of $[-1,1]^{2}$ such that $f( \pm 1, \pm 1)$ are finite and the norm

$$
\begin{aligned}
\|f\|_{L^{2}\left(\varpi_{\alpha, \gamma}, \varpi_{\beta, \gamma}\right)}= & \left(c_{\alpha, \gamma} \int_{-1}^{1}\left(|f(x, 1)|^{2}+|f(x,-1)|^{2}\right) \varpi_{\alpha, \gamma}(x) \mathrm{d} x\right. \\
& \left.+c_{\beta, \gamma} \int_{-1}^{1}\left(|f(1, y)|^{2}+|f(-1, y)|^{2}\right) \varpi_{\beta, \gamma}(y) \mathrm{d} y\right)^{\frac{1}{2}} .
\end{aligned}
$$

is finite for every $f$. For $f \in L^{2}\left([-1,1]^{2}, \varpi_{\alpha, \gamma}, \varpi_{\beta, \gamma}\right)$, its Fourier orthogonal expansion is defined by

$$
f=\sum_{n=0}^{2} \sum_{i=1}^{n+1} \widehat{f}_{n, i} Y_{n, i}^{\alpha, \beta, \gamma}+\sum_{n=3}^{\infty} \sum_{i=1}^{4} \widehat{f}_{n, i} Y_{n, i}^{\alpha, \beta, \gamma}, \quad \widehat{f}_{n, i}=\frac{\left\langle f, Y_{n, i}^{\alpha, \beta, \gamma}\right\rangle}{\left\langle Y_{n, i}, Y_{n, i}^{\alpha, \beta, \gamma}\right\rangle}
$$

For $n \geq 2$, let $S_{n}(f)$ denotes its $n$-th partial sum defined by

$$
S_{n} f=\sum_{k=0}^{2} \sum_{i=1}^{k+1} \widehat{f}_{k, i} Y_{k, i}^{\alpha, \beta, \gamma}+\sum_{k=3}^{n} \sum_{i=1}^{4} \widehat{f}_{k, i} Y_{k, i}^{\alpha, \beta, \gamma}
$$

For fixed $\alpha, \beta, \gamma$, let $\langle\cdot, \cdot\rangle_{\alpha+\delta_{1}, \beta+\delta_{2}, \gamma}$ be the inner product defined in the previous section with $\sigma=\sigma^{\alpha, \beta, \gamma}$. For $f$ defined on $[-1,1]^{2}$, we define four functions

$$
\begin{aligned}
& F_{e, e}(x, y)=\frac{1}{4}[f(x, y)+f(-x, y)+f(x,-y)+f(-x,-y)], \\
& F_{e, o}(x, y)=\frac{1}{4}[f(x, y)+f(-x, y)-f(x,-y)-f(-x,-y)], \\
& F_{o, e}(x, y)=\frac{1}{4}[f(x, y)-f(-x, y)+f(x,-y)-f(-x,-y)], \\
& F_{o, o}(x, y)=\frac{1}{4}[f(x, y)-f(-x, y)-f(x,-y)+f(-x,-y)],
\end{aligned}
$$

where the subindices indicate the parity of the function. For example, $F_{e, o}$ is even in $x$ variable and odd in $y$ variable. By definition,

$$
f(x, y)=F_{e, e}(x, y)+F_{e, o}(x, y)+F_{o, e}(x, y)+F_{o, o}(x, y) .
$$

We further define

$$
\begin{aligned}
& G_{0,0}(x, y)=F_{e, e}(x, y), \quad G_{0,1}(x, y)=y^{-1} F_{e, o}(x, y) \\
& G_{1,0}(x, y)=x^{-1} F_{o, e}(x, y), \quad G_{1,1}(x, y)=x^{-1} y^{-1} F_{o, o}(x, y)
\end{aligned}
$$

and define $\psi: \mathbb{R}^{2} \mapsto \mathbb{R}^{2}$ by $\psi:(x, y) \mapsto(\sqrt{x}, \sqrt{y})$. Changing variables in integrals as in (3.3), we see that if $f \in L^{2}\left([-1,1]^{2}, \varpi_{\alpha, \gamma}, \varpi_{\beta, \gamma}\right)$, then $G_{\delta_{1}, \delta_{2}} \circ \psi \in$ $L^{2}\left(\mathcal{B}, w_{\alpha+\delta_{1}, \gamma}, w_{\beta+\delta_{2}, \gamma}\right)$ for $\delta_{i} \in\{0,1\}$. 
Theorem 3.3 For $f \in L^{2}\left([-1,1]^{2}, \varpi_{\alpha, \gamma}, \varpi_{\beta, \gamma}\right)$,

$$
\begin{aligned}
S_{2 m} f(x, y)= & S_{m}^{\alpha, \beta, \gamma} G_{0,0} \circ \psi\left(x^{2}, y^{2}\right)+y S_{m-1}^{\alpha, \beta+1, \gamma} G_{0,1} \circ \psi\left(x^{2}, y^{2}\right) \\
& +x S_{m-1}^{\alpha+1, \beta, \gamma} G_{1,0} \circ \psi\left(x^{2}, y^{2}\right)+x y S_{m-1}^{\alpha+1, \beta+1, \gamma} G_{1,1} \circ \psi\left(x^{2}, y^{2}\right), \\
S_{2 m+1} f(x, y)= & S_{m}^{\alpha, \beta, \gamma} G_{0,0} \circ \psi\left(x^{2}, y^{2}\right)+y S_{m}^{\alpha, \beta+1, \gamma} G_{0,1} \circ \psi\left(x^{2}, y^{2}\right) \\
& +x S_{m}^{\alpha+1, \beta, \gamma} G_{1,0} \circ \psi\left(x^{2}, y^{2}\right)+x y S_{m-1}^{\alpha+1, \beta+1, \gamma} G_{1,1} \circ \psi\left(x^{2}, y^{2}\right) .
\end{aligned}
$$

In particular, the norm of $S_{n} f-f$ is bounded by those of $S_{m}^{\alpha+\delta_{1}, \beta+\delta_{2}, \gamma} G_{\delta_{1}, \delta_{2}}-G_{\delta_{1}, \delta_{2}}$ as in Theorem 2.9.

Proof Using the parity of the function, it is easy to see that

$$
\frac{\left\langle f, Y_{2 m, i}\right\rangle}{\left\langle Y_{2 m, i}, Y_{2 m, i}\right\rangle}=\frac{\left\langle F_{e, e}, Y_{2 m, i}\right\rangle}{\left\langle Y_{2 m, i}, Y_{2 m, i}\right\rangle}=\frac{\left\langle G_{0,0} \circ \psi, p_{2 m, i}^{\alpha, \beta, \gamma}\right\rangle_{\alpha, \beta, \gamma}}{\left\langle p_{2 m, i}^{\alpha, \beta, \gamma}, p_{2 m, i}^{\alpha, \beta, \gamma}\right\rangle_{\alpha, \beta, \gamma}}, \quad i=1,2
$$

where we have used the fact that $F_{e, e}$ is even in both variables and use the change of variables in integrals as in (3.3). The similar procedure can be used in the other three cases, as $G_{i}(x, y)$ is even in both variables, and the result is

$$
\begin{aligned}
\frac{\left\langle f, Y_{2 m, i}\right\rangle}{\left\langle Y_{2 m, i}, Y_{2 m, i}\right\rangle} & =\frac{\left\langle F_{o, o}, Y_{2 m, i}\right\rangle}{\left\langle Y_{2 m, i}, Y_{2 m, i}\right\rangle}=\frac{\left\langle G_{1,1} \circ \psi, p_{m, i}^{\alpha+1, \beta+1, \gamma}\right\rangle_{\alpha+1, \beta+1, \gamma}}{\left\langle p_{2 m, i}^{\alpha+1, \beta+1, \gamma}, p_{2 m, i}^{\alpha+1, \beta+1, \gamma}\right\rangle_{\alpha+1, \beta+1, \gamma}} \\
i & =3,4, \\
\frac{\left\langle f, Y_{2 m+1, i}\right\rangle}{\left\langle Y_{2 m+1, i}, Y_{2 m+1, i}\right\rangle} & =\frac{\left\langle F_{e, o}, Y_{2 m+1, i}\right\rangle}{\left\langle Y_{2 m+1, i}, Y_{2 m+1, i}\right\rangle}=\frac{\left\langle G_{0,1} \circ \psi, p_{m, i}^{\alpha, \beta+1, \gamma}\right\rangle_{\alpha, \beta+1, \gamma}}{\left\langle p_{2 m, i}^{\alpha, \beta+1, \gamma}, p_{2 m, i}^{\alpha, \beta+1, \gamma}\right\rangle_{\alpha, \beta+1, \gamma}} \\
\frac{\langle i}{\left\langle f, Y_{2 m+1, i}\right\rangle} & =\frac{\left\langle F_{o, e}, Y_{2 m+1, i}\right\rangle}{\left\langle Y_{2 m+1, i}, Y_{2 m+1, i}\right\rangle}=\frac{\left\langle G_{1,0} \circ \psi, p_{m, i}^{\alpha+1, \beta, \gamma}\right\rangle_{\alpha+1, \beta, \gamma}}{\left\langle p_{2 m, i}^{\alpha+1, \beta, \gamma}, p_{2 m, i}^{\alpha+1, \beta, \gamma}\right\rangle_{\alpha+1, \beta, \gamma}} \\
i & =3,4 .
\end{aligned}
$$

Since $S_{n}^{\alpha+\delta_{1}, \beta+\delta_{2}, \gamma} G_{\delta_{1}, \delta_{2}} \circ \psi\left(x^{2}, y^{2}\right) \rightarrow G_{\delta_{1}, \delta_{2}}(x, y)$ and

$$
f(x, y)=G_{0,0}(x, y)+y G_{0,1}(x, y)+y G_{1,0}(x, y)+x y G_{1,1}(x, y),
$$

the last statement is evident.

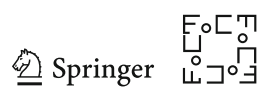




\section{Orthogonal System on the Square}

Let $w$ be a nonnegative weight function defined on $[0,1]$. Define

$$
W(x, y)=w(\max \{|x|,|y|\}), \quad(x, y) \in[-1,1]^{2} .
$$

We construct a system of orthogonal functions with respect to the inner product

$$
\langle f, \gamma\rangle_{W}=\int_{-1}^{1} \int_{-1}^{1} f(x, y) g(x, y) W(x, y) \mathrm{d} x \mathrm{~d} y .
$$

by making use of the orthogonal polynomials on the boundary or the square, studied in the previous section. Our starting point is the following integral identity derived from changing variables $(x, y) \mapsto(s \xi, s \eta)$,

$$
\int_{-1}^{1} \int_{-1}^{1} f(x, y) w(\max \{|x|,|y|\}) \mathrm{d} x \mathrm{~d} y=\int_{0}^{s} s \int_{\mathcal{B}} f(s \xi, s \eta) \mathrm{d} \sigma(\xi, \eta) w(s) \mathrm{d} s,
$$

where $\int_{\mathcal{B}} \mathrm{d} \sigma$ denotes the integral on the boundary of the square,

$$
\int_{\mathcal{B}} f(\xi, \eta) \mathrm{d} \sigma(\xi, \eta)=\int_{-1}^{1}[f(\xi, 1)+f(\xi,-1)] \mathrm{d} \xi+\int_{-1}^{1}[f(1, \eta)+f(-1, \eta)] \mathrm{d} \eta
$$

Our orthogonal functions are similar in structure to orthogonal polynomials on the unit disc that are constructed using spherical harmonics. However, these function are polynomials in $(s, \xi, \eta)$ for the $(x, y)=(s \xi, s \eta) \in[-1,1]^{2}$, but not polynomials in $(x, y)$.

Let $\mathcal{B} \mathcal{V}_{n}^{2}$ be the space of orthogonal polynomials on the boundary of $[-1,1]^{2}$ with respect to the inner product

$$
\langle f, g\rangle=\int_{\mathcal{B}} f(\xi, \eta) g(\xi, \eta) \mathrm{d} \sigma(\xi, \eta)
$$

which is the inner product with $\alpha=-\frac{1}{2}, \beta=-\frac{1}{2}$ and $\gamma=0$ studied in the previous section. Let $Y_{n, i}$ be an orthogonal basis for $\mathcal{B} \mathcal{V}_{n}^{2}$. For $n \leq 2$, they are defined by, see Theorem 3.2,

$$
\begin{aligned}
& Y_{0,1}(x, y)=1, \quad Y_{1,1}(x, y)=x, \quad Y_{1,2}(x, y)=y ; \\
& Y_{2,1}(x, y)=x^{2}-\frac{2}{3}, \quad Y_{2,2}(x, y)=x y, \quad Y_{2,3}^{2}(x, y)=y^{2}-\frac{2}{3},
\end{aligned}
$$

whereas for $n \geq 3$, they are constructed in Theorem 3.2. For $n \geq k$, denote by $P_{m, 2 n-2 k}$ the orthogonal polynomial of degree $m$ with respect to $t^{2 n-2 k+1} w(t)$ on $[0,1]$ and with $P_{0,2 n-2 k}(s):=1$. For $n \geq 0$ and $0 \leq k \leq n$, we define 


$$
Q_{k, i}^{n}(x, y):=P_{k, 2 n-2 k}(s) s^{n-k} Y_{n-k, i}\left(\frac{\xi}{s}, \frac{\eta}{s}\right)
$$

where $i=1, \ldots, \min \{n+1,4\}$.

Theorem 4.1 In the coordinates $(x, y)=s(\xi, \eta)$, the system of functions

$$
\left\{Q_{k, i}^{n}: i=1, \ldots, \min \{n+1,4\}, 0 \leq k \leq n, n \geq 0\right\}
$$

is a complete orthogonal basis for $L^{2}\left(W ;[-1,1]^{2}\right)$.

Proof Changing variables $x=s \xi$ and $y=\eta$ shows

$$
\begin{aligned}
\left\langle Q_{k, i}^{n}, Q_{l, j}^{m}\right\rangle_{W}= & \int_{0}^{1} P_{k, 2 n-2 k}(s) P_{l, 2 m-2 l}(s) s^{n-k+m-l+1} w(s) \mathrm{d} s \\
& \times \int_{\mathcal{B}} Y_{n-k, i}(\xi, \eta) Y_{m-l, j}(\xi, \eta) \mathrm{d} \sigma(\xi, \eta) .
\end{aligned}
$$

The second integral is zero if $i \neq j$ and $n-k \neq m-l$, whereas the second integral is zero when $n-k=m-l$ and $k \neq l$, so that $\left\langle Q_{k, i}^{n}, Q_{l, j}^{m}\right\rangle_{W}=0$ if $i \neq j, k \neq l$ and $n \neq m$. By definition, $s^{n-k} Y_{n-k, i}\left(\frac{\xi}{s}, \frac{\eta}{s}\right)$ is a polynomial of degree $n-k$ in the variable $s$, so that $Q_{k, i}^{n}$ is a polynomial of degree $n$. To show that the system is complete, we show that if $\left\langle f, Q_{k, i}^{n}\right\rangle=0$ for all $k, i, n$, then $f(x, y)=0$. Indeed, by the orthogonality of polynomials on the boundary, we see that

$$
\begin{aligned}
f(x, y)=f(s \xi, s \eta) & =\sum_{k=0}^{n} s^{k} \sum_{j=0}^{k} a_{j, k} \xi^{j} \eta^{k-j} \\
& =\sum_{k=0}^{n} s^{k} \sum_{m=0}^{k} \sum_{i=1}^{\min \{m+1,4\}} b_{m, i}^{k} Y_{m, i}(\xi, \eta)
\end{aligned}
$$

modulo the ideal. Changing order of summation shows that

$$
\begin{aligned}
f(x, y) & =\sum_{m=0}^{n} \sum_{i=1}^{\min \{m+1,4\}}\left(\sum_{k=0}^{n-m} b_{m, i}^{k+m} s^{k}\right) s^{m} Y_{m, i}(\xi, \eta) \\
& =\sum_{m=0}^{n} \sum_{i=1}^{\min \{m+1,4\}}\left(\sum_{k=0}^{n-m} c_{m, i, k} P_{k, 2 m}(x)\right) s^{m} Y_{m, i}(\xi, \eta) .
\end{aligned}
$$

This completes the proof.

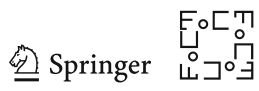




\section{Sampling the Associated Determinantal Point Process}

Associated with an orthonormal basis $q_{0}(x), \ldots, q_{N}(x)$ is a determinantal point process, which describes $N$ points $\lambda_{1}, \ldots, \lambda_{N}$ distributed according to

$$
\operatorname{det}\left(\begin{array}{ccc}
K_{N}\left(\lambda_{1}, \lambda_{1}\right) & \cdots & K_{N}\left(\lambda_{1}, \lambda_{N}\right) \\
\vdots & \ddots & \vdots \\
K_{N}\left(\lambda_{N}, \lambda_{1}\right) & \cdots & K_{N}\left(\lambda_{N}, \lambda_{N}\right)
\end{array}\right)
$$

where

$$
K_{N}(x, y)=\sum_{k=0}^{N} q_{k}(x) q_{k}(y)
$$

is the reproducing kernel, see [1] for an overview of determinantal point processes.

In the particular case of univariate orthogonal polynomials with respect to a weight $w(x)$, the associated determinantal process is equivalent to a Coulomb gas - that is, the points are distributed according to

$$
\frac{1}{Z_{N}} \prod_{k=1}^{N} w\left(x_{k}\right) \prod_{k<j}\left|\lambda_{k}-\lambda_{j}\right|^{2}
$$

where $Z_{N}$ is the normalization constant—as well as the eigenvalues of unitary ensembles, see for example [3] for the case of an analytic weight on the real line or [8] for the case of a weight supported on $[-1,1]$ with Jacobi-like singularities.

In the case of our orthogonal polynomials on the wedge, the connection with Coulomb gases and random matrix theory is no longer obvious: the interaction of the points is not Coulomb (that is, it cannot be reduced to a Vandermonde determinant squared times a product of weights), nor is there an obvious distribution of random matrices whose eigenvalues are associated with the points. ${ }^{1}$ We note that there are recent universality results due to Kroó and Lubinsky on the asymptotics of Christoffel functions associated with multivariate orthogonal polynomials [6,7], but they do not apply in our setting.

Using the algorithm for sampling determinantal point processes associated with univariate orthogonal polynomials [10], which is trivially adapted to the orthogonal polynomials on the wedge, we can sample from this determinantal point process. We use this algorithm to calculate statistics of the points. In Fig. 1, we use the sampling algorithm in a Monte Carlo simulation to approximate the probability that no eigenvalue is present in a neighbourhood of three points for $\alpha=\beta=\gamma=0$. That is, we take 10,000 samples of a determinantal point process, and calculate the distance of the nearest point to $z_{0}$, for $z_{0}$ equal to $(1,1),(0,1),(0.5,1)$ and $(0.7,1)$. The plots are of a complementary empirical cumulative distribution function of these samples.

\footnotetext{
1 If there is such a random matrix distribution, one would expect it to be a pair of commuting random matrices, whose joint eigenvalues give points on the wedge.
} 

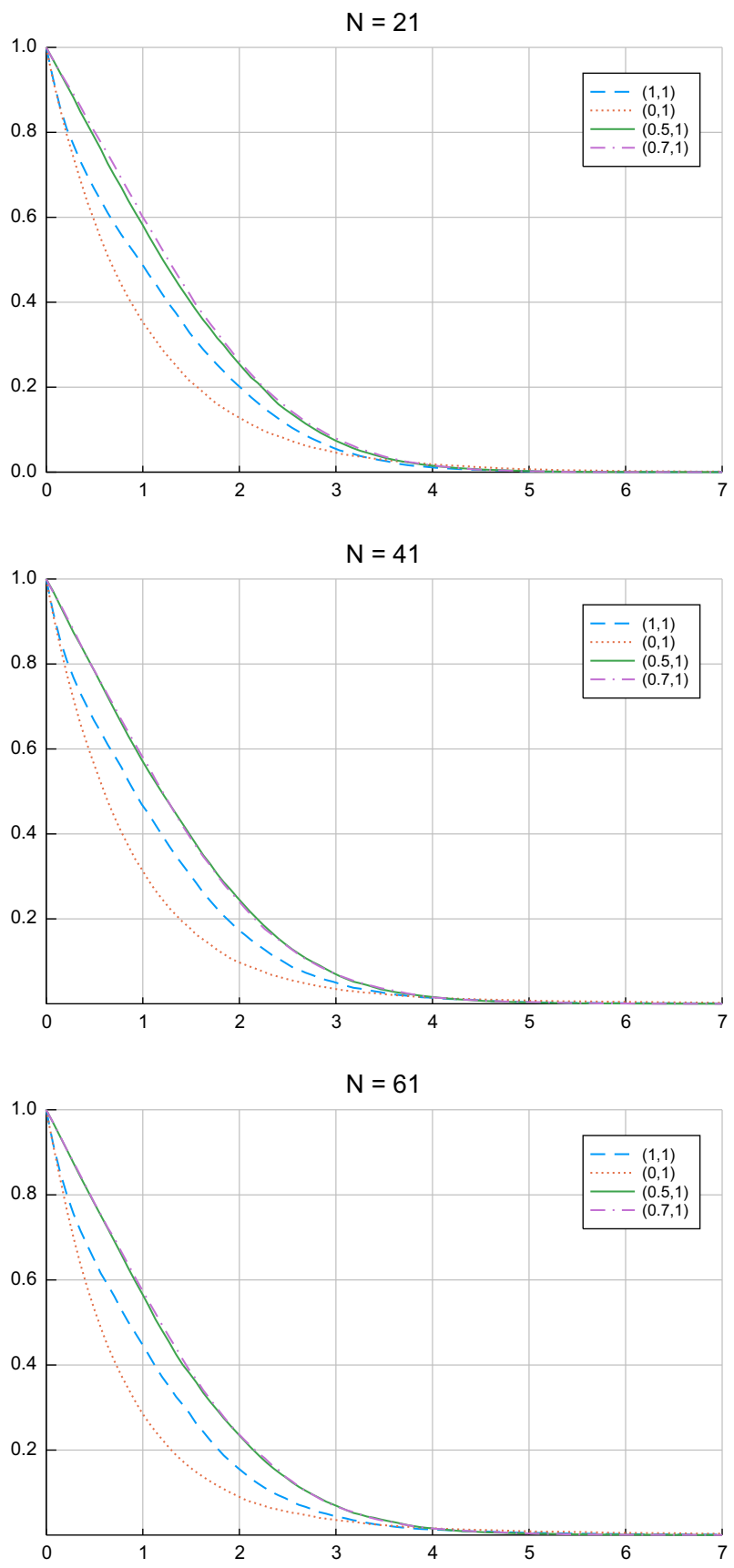

Fig. 1 Monte Carlo calculation of the probability that no point satisfying $y=1$ sampled according to the determinantal point process associated to the wedge orthogonal polynomials with $\alpha=\beta=\gamma=0$ lies in a neighbourhood of four different points. $N$ is the total number of basis elements and points. We have scaled the statistics so that the variance is one, and have used 10,000 samples 

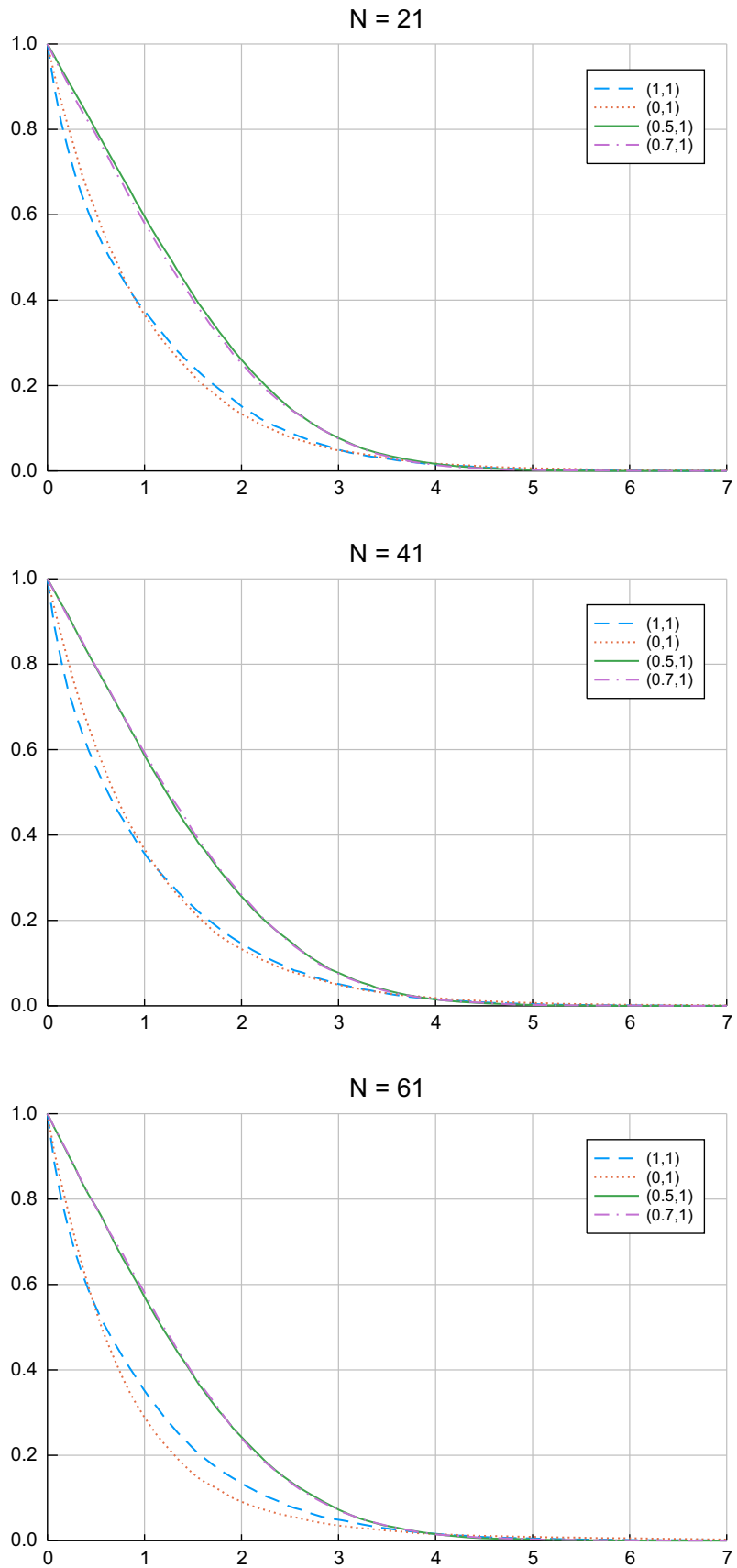

Fig. 2 Monte Carlo calculation of the probability that no point satisfying $y=1$ sampled according to the Coulomb gas on the wedge lies in a neighbourhood of four different points. $N$ is the total number of basis elements and points. We have scaled the statistics so that the variance is one, and have used 10,000 samples 


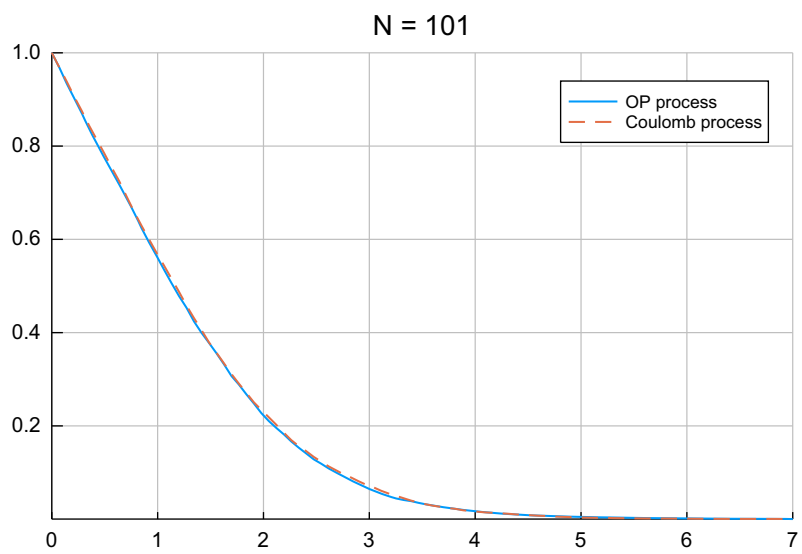

Fig. 3 Comparison of the gap probability of the determinantal point process associated to the wedge orthogonal polynomials and Coulomb gas near $(0.5,1)$ for $N=101$ points. We have scaled the statistics so that the variance is one, and have used 10,000 samples

This gives an estimation of the probability that no eigenvalue is in a neighbourhood of $z_{0}$. We have scaled the distributions so that the empirical variance is one: this ensures that the distributions tend to a limit as $N$ becomes large, which is the regime where universality is present.

In Fig. 2 we plot the same statistics but for samples from the unweighted Coulomb gas on the wedge, which has the distribution

$$
\frac{1}{Z_{N}} \prod_{k<j}\left\|\lambda_{k}-\lambda_{j}\right\|^{2}
$$

for $\lambda_{k}$ supported on the wedge. As this is a Vandermonde determinant squared, it is also a determinantal point process with the basis arising from orthogonalized complexvalued polynomials $1,(x+i y),(x+i y)^{2}, \ldots$ [2]. We approximate this orthogonal basis using the modified Gram-Schmidt algorithm with the wedge inner product calculated via Clenshaw-Curtis quadrature. Again, this fits naturally into the sampling algorithm of [10], hence we can produce samples of this point process. What we observe is that, while our determinantal point process is not a Coulomb gas, it appears to be in the same universality class as the Coulomb gas away from the edge and corner, as the statistics follow the same distribution. This universality class matches that of the Gaussian Unitary Ensemble, as seen in Fig. 3 where we compare the three for $N=50$.

\section{Conclusion}

We have introduced multivariate orthogonal polynomials on the wedge and boundary of a square for some natural choices of weights. We have also generated a complete orthogonal basis with respect to a suitable weight inside the square. We have looked at determinantal point process statistics and observed a relationship between the resulting

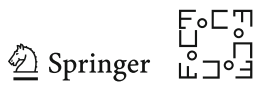


statistics and Coulomb gases, suggesting that, away from the corner and edge, they are in the same universality class.

One of the motivations for this work is to solve singular integral equations and evaluate their solutions on contours that have corners, in other words, to generalized the approach of [11]. Preliminary work in this direction is included in "Appendix B", which shows how the recurrence relationship that our polynomials satisfy can be used to evaluate Stieltjes transforms.

Open Access This article is distributed under the terms of the Creative Commons Attribution 4.0 International License (http://creativecommons.org/licenses/by/4.0/), which permits unrestricted use, distribution, and reproduction in any medium, provided you give appropriate credit to the original author(s) and the source, provide a link to the Creative Commons license, and indicate if changes were made.

\section{Appendix A. Jacobi Operators}

By necessity, multivariate orthogonal polynomials have block-tridiagonal Jacobi operators corresponding to multiplication by $x$ and $y$. We include here the recurrences associated with the inner product $\langle f, g\rangle_{\alpha, \alpha, \gamma}$ (that is, $\beta=\alpha$ ) that encode the Jacobi operators as they have a particularly simple form. The following lemma gives a linear combination of our orthogonal polynomials that vanish on $x=1$ :

Proposition A.1 For $\beta=\alpha$, we have

$$
(\alpha+\gamma+2) Q_{1}(x, y)-P_{1}(x, y)+(1+\alpha) P_{0}(x, y)=2(\alpha+\gamma+2)(1-x)
$$

and for $n=1,2, \ldots$,

$$
\begin{aligned}
& (n+\gamma+\alpha+2) Q_{n+1}(x, y)-(n+1) P_{n+1}(x, y)-(n+\gamma) Q_{n}(x, y) \\
& \quad+(n+a+1) P_{n}(x, y) \\
& \quad=2(1-x)(2 n+\gamma+\alpha+2) P_{n}^{(\gamma+1, \alpha)}(2 x-1)
\end{aligned}
$$

Proposition A.2 Assume $(1-x)(1-y)=0$. Then

$$
\begin{aligned}
& (1-x) P_{0}(x, y)=\frac{1}{2} Q_{1}(x, y)-\frac{1}{2(2+\gamma+\alpha)} P_{1}(x, y)+\frac{1+\gamma}{2(2+\gamma+\alpha)} P_{0}(x, y) \\
& (1-x) P_{1}(x, y)=\frac{\gamma+\alpha+2}{2(4+\gamma+\alpha)} Q_{2}(x, y)-\frac{\gamma+\alpha+2}{(3+\gamma+\alpha)(4+\gamma+\alpha)} P_{2}(x, y) \\
& -\frac{1+\alpha}{4+\gamma+\alpha} Q_{1}(x, y)+\frac{4+3 \alpha+\gamma(3+\gamma+\alpha)}{2(2+\gamma+\alpha)(4+\gamma+\alpha)} P_{1}(x, y) \\
& -\frac{(1+\gamma)(1+\alpha)}{2(2+\gamma+\alpha)(3+\gamma+\alpha)} P_{0}(x, y) \\
& (1-x) Q_{1}(x, y)=-\frac{1}{2(4+\gamma+\alpha)} Q_{2}(x, y)+\frac{1}{(3+\gamma+\alpha)(4+\gamma+\alpha)} P_{2}(x, y)
\end{aligned}
$$




$$
\begin{aligned}
& +\frac{(3+\gamma)}{2(4+\gamma+\alpha)} Q_{1}(x, y)-\frac{(2+\gamma)}{(2+\gamma+\alpha)(4+\gamma+\alpha)} P_{1}(x, y) \\
& +\frac{(1+\gamma)(2+\gamma)}{2(2+\gamma+\alpha)(3+\gamma+\alpha)} P_{0}(x, y)
\end{aligned}
$$

and, for $n=2,3, \ldots$,

$$
\begin{aligned}
& (1-x) P_{n}(x, y)=\frac{(1+\gamma+\alpha+n)(n+\gamma+\alpha+2)}{2(1+\gamma+\alpha+2 n)(2+\gamma+\alpha+2 n)} Q_{n+1}(x, y) \\
& -\frac{(1+\gamma+\alpha+n)(n+1)}{2(1+\gamma+\alpha+2 n)(2+\gamma+\alpha+2 n)} P_{n+1}(x, y) \\
& -\frac{(\alpha+n)(1+\gamma+\alpha+n)(1+\gamma+\alpha+2 n)}{(1+\gamma+\alpha+2 n)(2+\gamma+\alpha+2 n)(\gamma+\alpha+2 n)} Q_{n}(x, y) \\
& +\frac{(1+\gamma)(\gamma+\alpha)+2(1+\gamma+\alpha) n+2 n^{2}}{2(2+\gamma+\alpha+2 n)(\gamma+\alpha+2 n)} P_{n}(x, y) \\
& +\frac{(n+\alpha)(n+\alpha-1)}{2(1+\gamma+\alpha+2 n)(2 n+\gamma+\alpha)} Q_{n-1}(x, y) \\
& -\frac{(n+\alpha)(n+\gamma)}{2(1+\gamma+\alpha+2 n)(2 n+\gamma+\alpha)} P_{n-1}(x, y) \\
& (1-x) Q_{n}(x, y)=-\frac{n(2+\gamma+\alpha+n)}{2(2+\gamma+\alpha+2 n)(2 n+\gamma+\alpha+1)} Q_{n+1}(x, y) \\
& +\frac{n(1+n)}{2(2+\gamma+\alpha+2 n)(2 n+\gamma+\alpha+1)} P_{n+1}(x, y) \\
& +\frac{(1+\gamma)(2+\gamma+\alpha)+2(1+\gamma+\alpha) n+2 n^{2}}{2(\gamma+\alpha+2 n)(2+\gamma+\alpha+2 n)} Q_{n}(x, y) \\
& -\frac{n(1+\gamma+n)}{(\gamma+\alpha+2 n)(2+\gamma+\alpha+2 n)} P_{n}(x, y) \\
& -\frac{(1+\gamma+n)(\alpha+n-1)}{2(\gamma+\alpha+2 n)(2 n+\gamma+\alpha+1)} Q_{n-1}(x, y) \\
& \frac{(1+\gamma+n)(\gamma+n)}{2(\gamma+\alpha+2 n)(2 n+\gamma+\alpha+1)} P_{n-1}(x, y)
\end{aligned}
$$

Proof The first equation follows from Proposition A.1, since, for $y=1$, we have (using $[9,(18.9 .5)]$ to increment the first parameter)

$$
\begin{aligned}
(2 n+\gamma+\alpha+1)(1-x) P_{n}(x, y) & =(n+\gamma+\alpha+1)(1-x) P_{n}^{(\gamma+1, \alpha)}(2 x-1) \\
& -(n+\alpha)(1-x) P_{n-1}^{(\gamma+1, \alpha)}(2 x-1)
\end{aligned}
$$

The second equation also follows from Proposition A.1, since, for $y=1$, we have (using [9, (18.9.6)] to decrement the first parameter)

$$
(2 n+\gamma+\alpha+1)(1-x) Q_{n}(x, y)=-n(1-x) P_{n}^{(\gamma+1, \alpha)}(2 x-1)
$$




$$
+(n+\gamma+1)(1-x) P_{n-1}^{(\gamma+1, \alpha)}(2 x-1) .
$$

The recurrences for multiplication by $1-y$ follow from the symmetries $P_{n}(x, y)=$ $P_{n}(y, x)$ and $Q_{n}(x, y)=-Q_{n}(y, x)$.

\section{Appendix B. Stieltjes Transform of Orthogonal Polynomials}

Consider the Stieltjes transform

$$
\mathcal{S}_{\Omega} f(z)=\int_{\Omega} \frac{f(x, y)}{z-(x+i y)} \mathrm{d} s,
$$

where $\mathrm{d} s$ is the arc-length differential. Just as in one-dimensions, the Stieltjes transform of weighted multivariate orthogonal polynomials satisfies the same recurrence as the orthogonal polynomials themselves.

Proposition B.1 Suppose $\mathbb{P}_{n}$ are a family of orthogonal polynomials with respect to $w(x, y)$. Then, for $n=1,2, \ldots$,

$$
z \mathcal{S}_{\Omega}\left[\mathbb{P}_{n} w\right](z)=\mathcal{S}_{\Omega}\left[\zeta \mathbb{P}_{n} w\right](z)
$$

In particular, if $\mathbb{P}_{n}$ satisfies the recurrence relationships

$$
\begin{aligned}
& x \mathbb{P}_{n}=C_{n}^{x} \mathbb{P}_{n-1}+A_{n}^{x} \mathbb{P}_{n}+B_{n}^{x} \mathbb{P}_{n+1} \\
& y \mathbb{P}_{n}=C_{n}^{y} \mathbb{P}_{n-1}+A_{n}^{y} \mathbb{P}_{n}+B_{n}^{y} \mathbb{P}_{n+1}
\end{aligned}
$$

then for $A_{n}^{z}=A_{n}^{x}+\mathrm{i} A_{n}^{y}, B_{n}^{z}=B_{n}^{x}+\mathrm{i} B_{n}^{y}$ and $C_{n}^{z}=C_{n}^{x}+\mathrm{i} C_{n}^{y}$ we have

$$
z \mathcal{S}_{\Omega}\left[\mathbb{P}_{n} w\right](z)=C_{n}^{z} \mathcal{S}_{\Omega}\left[\mathbb{P}_{n-1} w\right](z)+A_{n}^{z} \mathcal{S}_{\Omega}\left[\mathbb{P}_{n} w\right](z)+B_{n}^{z} \mathcal{S}_{\Omega}\left[\mathbb{P}_{n+1} w\right](z)
$$

Proof We will identify $\mathbb{R}^{2}$ and $\mathbb{C}$ and use the notation $\zeta=x+i y$. Note that

$$
z \int_{\Omega} \frac{f(\zeta)}{z-\zeta} \mathrm{d} s=\int_{\Omega} \frac{(z-\zeta) f(\zeta)}{z-\zeta} \mathrm{d} s+\int_{\Omega} \frac{\zeta f(\zeta)}{z-\zeta} \mathrm{d} s=\int_{\Omega} f(\zeta) \mathrm{d} s+\mathcal{S}_{\Omega}[\zeta f](z)
$$

The first integral is zero if $f$ is orthogonal to 1 .

While this holds true for all families of multivariate orthogonal polynomials, in general, satisfying a single recurrence is not sufficient to determine $\mathcal{S}_{\Omega}\left[\mathbb{P}_{n} w\right](z)$. However, since our blocks are square, in our case it is:

Corollary B.2 If $B_{n}^{z}=B_{n}^{x}+\mathrm{i} B_{n}^{y}$ is invertible, then

$$
\begin{aligned}
\mathcal{S}_{\Omega}\left[\mathbb{P}_{n+1} w\right](z)= & z\left(B_{n}^{z}\right)^{-1} \mathcal{S}_{\Omega}\left[\mathbb{P}_{n} w\right](z)-\left(B_{n}^{z}\right)^{-1} C_{n}^{z} \mathcal{S}_{\Omega}\left[\mathbb{P}_{n-1} w\right](z)-\left(B_{n}^{z}\right)^{-1} \\
& \times A_{n}^{z} \mathcal{S}_{\Omega}\left[\mathbb{P}_{n} w\right](z)
\end{aligned}
$$


This means that we can calculate the Stieltjes transform in linear time by solving the recurrence equation, using explicit formulas for the $n=0$ and $n=1$ terms. Unfortunately, the results are numerically unstable for both $z$ on and off the contour. Here we sketch an alternative approach built on (F.W.J.) Olver's and Miller's algorithm, see [9, Section 3.6] for references in the tridiagonal setting and [5, Section 2.3] for the equivalent application to calculating Stieltjes transforms of univariate orthogonal polynomials.

For $z$ off the contour, we can successfully and stably calculate the Stieltjes transform using a block-wise version of Olver's algorithm, which is equivalent to solving the $2 n+1 \times 2 n+1$ block-tridiagonal system

$$
\begin{aligned}
\mathbf{q}_{0} & =1 \\
C_{k}^{z} \mathbf{q}_{k-1}+\left(A_{k}^{z}-z_{k} I\right) \mathbf{q}_{k}+B_{k}^{z} \mathbf{q}_{k+1} & =0 \quad \text { for } k=1,2, \ldots, n-1 \\
\mathbf{q}_{n} & =\left(\begin{array}{l}
0 \\
0
\end{array}\right)
\end{aligned}
$$

where $\mathbf{q}_{0} \in \mathbb{C}^{1}$ and $\mathbf{q}_{k} \in \mathbb{C}^{2}$ for $k=1,2, \ldots, n$. Then

$$
\mathcal{S}_{\Omega}\left[\mathbb{P}_{k} w\right](z) \approx \mathcal{S}_{\Omega}\left[\mathbb{P}_{0} w\right](z) \mathbf{q}_{k}
$$

Olver's algorithm consists of performing Gaussian elimination adaptively until a convergence criteria is satisfied.

For $z$ on or near the contour, we no longer see quick decay in the Stieltjes transform (it is no longer a minimal solution to the recurrence), hence $n$ must be prohibitively large. Instead, we adapt Olver's algorithm in a vein similar to Miller's algorithm to allow for a non-decaying tail. We do so by calculating two additional solutions $\mathbf{q}^{1}$, and $\mathbf{q}^{2}$ (with the same block-sizes as before) satisfying:

$$
\begin{gathered}
\mathbf{q}_{0}^{j}=1 \\
C_{k}^{z} \mathbf{q}_{k-1}^{j}+\left(A_{k}^{z}-z I\right) \mathbf{q}_{k}^{j}+B_{k}^{z} \mathbf{q}_{k+1}^{j}=0 \\
\mathbf{q}_{n}^{1}=\left(\begin{array}{l}
1 \\
0
\end{array}\right) \quad \text { and } \quad \mathbf{q}_{n}^{2}=\left(\begin{array}{l}
0 \\
1
\end{array}\right) .
\end{gathered}
$$

These three solutions avoid picking up the exponentially growing solution that forward recurrence does. Thus we can solve a $3 \times 3$ system for constants $a, b$ and $c$ satisfying

$$
\begin{aligned}
& a(z) \mathbf{q}_{0}+b(z) \mathbf{q}_{0}^{1}+c(z) \mathbf{q}_{0}^{2}=\mathcal{S}_{\Omega}\left[\mathbb{P}_{0} w\right](z) \\
& a(z) \mathbf{q}_{1}+b(z) \mathbf{q}_{1}^{1}+c(z) \mathbf{q}_{1}^{2}=\mathcal{S}_{\Omega}\left[\mathbb{P}_{1} w\right](z)
\end{aligned}
$$

We immediately have that

$$
\mathcal{S}_{\Omega}\left[\mathbb{P}_{k} w\right](z)=a(z) \mathbf{q}_{k}+b(z) \mathbf{q}_{k}^{1}+c(z) \mathbf{q}_{k}^{2} \quad \text { for } \quad k=0,1, \ldots, n .
$$




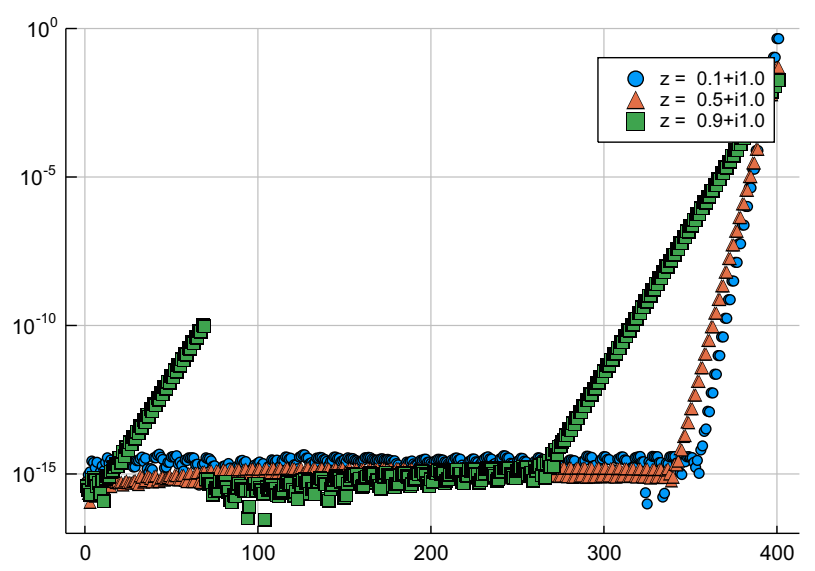

Fig. 4 Numerical error in approximating the Stieltjes transform on the wedge, with $\alpha=\beta=\gamma=0$ using (B.2). Note that we need to choose $n$ larger than necessary to avoid the errors in the tail, and there are unresolved numerical errors if $z$ is close to the corner

While this holds true for all $n$, we note that in practice we need to choose $n$ bigger than the number of coefficients in order to observe numerical stability, see Fig. 4. We also find that there are still stability issues near the corner. Resolving these issues is ongoing research.

\section{References}

1. A. Borodin, Chapter 11: Determinantal Point Processes, in The Oxford Handbook of Random Matrix Theory, eds. G. Akemann, J. Baik, P. Di Francesco, Oxford University Press, 2011

2. L.-L. Chau and O. Zaboronsky, On the structure of correlation functions in the normal matrix model, Comm. Math. Phys., 196(1) (1998), 203-247

3. P. Deift, Orthogonal Polynomials and Random Matrices: a Riemann-Hilbert Approach, American Mathematical Soc., 1999

4. C. F. Dunkl and Y. Xu, Orthogonal Polynomials of Several Variables, 2nd ed. Encyclopedia of Mathematics and its Applications 155, Cambridge University Press, 2014.

5. W. Gautschi, Orthogonal Polynomials: Computation and Approximation, Oxford University Press, 2004

6. A. Kroó and D. Lubinsky, Christoffel Functions and Universality on the Boundary of the Ball, Acta Math. Hungarica, 140 (2013), 117-133

7. A. Kroó and D. Lubinsky, Christoffel Functions and Universality in the Bulk for Multivariate Orthogonal Polynomials, Can. J. Maths, 65 (2013), 600-620

8. A. B. J. Kuijlaars and M. Vanlessen, Universality for eigenvalue correlations from the modified Jacobi unitary ensemble, Int. Maths Res. Not. 2002.30 (2002) 1575-1600

9. F. W. J. Olver, A. B. Olde Daalhuis, D. W. Lozier, B. I. Schneider, R. F. Boisvert, C. W. Clark, B. R. Miller, and B. V. Saunders, eds. NIST Digital Library of Mathematical Functions. http://dlmf.nist. gov/, Release 1.0.16 of 2017-09-18.

10. S. Olver, N. Raj Rao and T. Trogdon, Sampling unitary ensembles, Rand. Mat.: Th. Appl., 4 (2015) 1550002

11. R.M. Slevinsky amd S. Olver, A fast and well-conditioned spectral method for singular integral equations, J. Comp. Phys., 332 (2017) 290-315

12. G. Szegő, Orthogonal Polynomials, 4th edition, Amer. Math. Soc., Providence, RI. 1975. 